\title{
An Ontology for Human-Human Interactions and Learning Interaction Behavior Policies
}

\author{
OZGUR S. OGUZ, Technical University of Munich, Germany \\ WOLFGANG RAMPELTSHAMMER, University of Twente, The Netherlands \\ SEBASTIAN PAILLAN and DIRK WOLLHERR, Technical University of Munich, Germany
}

\begin{abstract}
Robots are expected to possess similar capabilities that humans exhibit during close proximity dyadic interaction. Humans can easily adapt to each other in a multitude of scenarios, ensuring safe and natural interaction. Even though there have been attempts to mimic human motions for robot control, understanding the motion patterns emerging during dyadic interaction has been neglected. In this work, we analyze close-proximity human-human interaction and derive an ontology that describes a broad range of possible interaction scenarios by abstracting tasks and using insights from attention theory. This ontology enables us to group interaction behaviors into separate cases, each of which can be represented by a particular graph. Using imitation learning, we train unique interaction policies with recurrent neural networks for each case. The ontology offers a unified and generic approach to categorically analyze and learn close-proximity interaction behaviors that can both be utilized as base models for future studies and enhance natural human-robot collaboration.
\end{abstract}

CCS Concepts: • Human-centered computing $\rightarrow$ Collaborative interaction; Interaction design theory, concepts and paradigms; $\bullet$ Computing methodologies $\rightarrow$ Robotic planning;

Additional Key Words and Phrases: Human-human interaction, ontology, behavior modeling, classification, imitation learning, human-robot interaction

\section{ACM Reference format:}

Ozgur S. Oguz, Wolfgang Rampeltshammer, Sebastian Paillan, and Dirk Wollherr. 2019. An Ontology for Human-Human Interactions and Learning Interaction Behavior Policies. ACM Trans. Hum.-Robot Interact. 8, 3, Article 16 (July 2019), 26 pages.

https://doi.org/10.1145/3326539

\section{INTRODUCTION}

As robots become ubiquitous in many aspects of daily life, e.g., in service robotics or manufacturing, close interaction with humans becomes a necessary functionality. Humans easily interact in shared and confined workspaces. They adapt to different interaction behaviors without compromising safety and efficiency (Figure 1). Especially in manufacturing, effective interaction in a shared workspace can increase productivity and, hence, is a desirable capability for robots. However, in contrast to humans, robots still need to have a safety distance from human collaborators

Authors' addresses: O. S. Oguz, Technical University of Munich, Electrical and Computer Engineering, Theresienstr. 90, Munich, 80333, Germany; email: o.oguz@tum.edu; W. Rampeltshammer, University of Twente, Biomechanical Engineering, Enschede, The Netherlands; email: w.f.rampeltshammer@utwente.nl; S. Paillan and D. Wollherr, Technical University of Munich, Electrical and Computer Engineering, Theresienstr. 90, Munich, 80333, Germany; emails: \{s.paillan, dw\}@tum.edu. Permission to make digital or hard copies of all or part of this work for personal or classroom use is granted without fee provided that copies are not made or distributed for profit or commercial advantage and that copies bear this notice and the full citation on the first page. Copyrights for components of this work owned by others than the author(s) must be honored. Abstracting with credit is permitted. To copy otherwise, or republish, to post on servers or to redistribute to lists, requires prior specific permission and/or a fee. Request permissions from permissions@acm.org.

(C) 2019 Copyright held by the owner/author(s). Publication rights licensed to ACM.

2573-9522/2019/07-ART16

https://doi.org/10.1145/3326539 

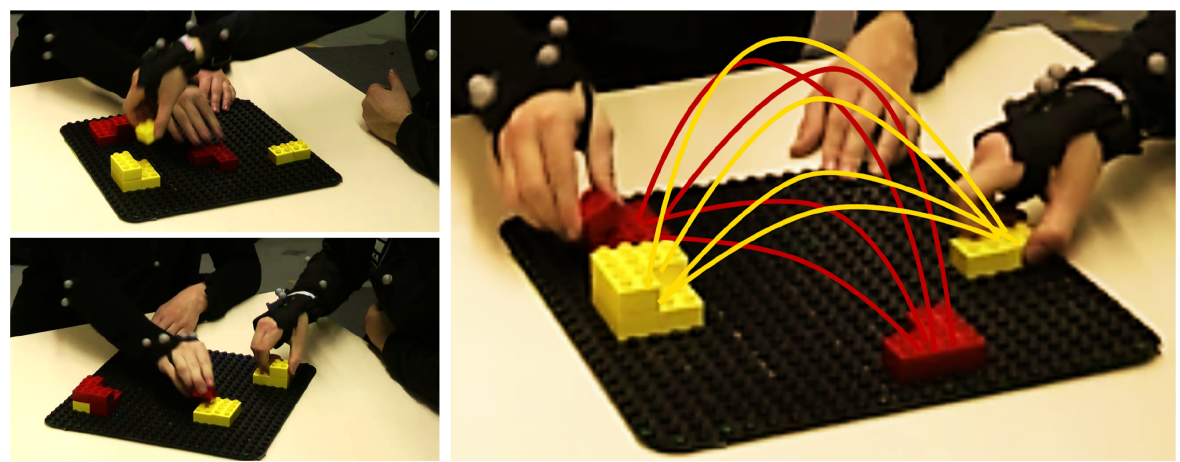

Fig. 1. Three different cases for close proximity human-human interaction scenarios, in which two collaborators need to adapt to each other's arm motion depending on the case. The possible options for the collaborators are showcased with sample trajectories (right).

due to their incapability to understand human interactions. How can we achieve a non-verbal communication directly through movement that results in a natural and seamless interaction between humans and robots? For such an intuitive human-robot interaction (HRI), the robot has to (i) accurately predict the human motion, and (ii) execute a natural responsive behavior, such that the human collaborator can easily predict the motion of the robot and adapt to it. Human movement features and the corresponding control policies during dyadic interaction can provide useful insights, since humans easily fulfill these two requirements (Sebanz and Knoblich 2009). In that regard, human-human interaction (HHI) studies are crucial to get a thorough understanding of interaction behaviors. In this work, we focus on upper body motions, specifically where the shared workspace imposes interfering arm reaching movements by the partners. Our aim is to understand $\mathrm{HHI}$ in a shared workspace by developing an ontology framework that defines the types and properties of close proximity interactions, as well as the relationships between them. Such a descriptive model can also be transferred to HRI scenarios to provide a seamless dyadic interaction.

There have been studies in neuroscience and psychology that investigated important factors for HHI. However, these studies, mainly on visual attention (Corbetta and Shulman 2002), synchronicity (Sebanz and Knoblich 2009), and motion prediction (Knoblich and Flach 2001) have specific task setups and have rarely investigated interaction in a shared workspace with complex tasks. A recent study extensively described and analyzed the factors influencing HHI (Sebanz and Knoblich 2009). However, a systematical analysis of HHI in close proximity from the perspective of human motion behavior is still missing. In addition to descriptive analysis, there have been studies that predict and imitate human motions for predefined specific setups (Hayne et al. 2016; Oguz et al. 2018b). A recent work by Mainprice et al. (2016) accurately generates motions for an HHI scenario, which were comparable to real human motions. However, it remains unclear whether their approach generalizes toward other setups and dyad positioning. Evidently, the challenges for describing and modeling HHI have been tackled in several fields in isolation. A comprehensive analysis and methods to define and learn models that generalize interaction behaviors have been neglected.

For an exhaustive analysis of close proximity HHI, the key factors from the aforementioned fields of research have to be considered together. Therefore, a generic interaction model needs to be developed that integrates insights from psychology and neuroscience, while generalizing toward a broad range of tasks. Such a formulation facilitates a systematic analysis of human interaction behaviors and enables the construction of a high-level representation of HHI scenarios. This descriptive representation can be used to classify interaction behaviors by analyzing movement 
related features (e.g., distance metrics). It is then possible to not only learn dyadic interaction policies from $\mathrm{HHI}$ demonstrations, but also predict human motions during interaction. By using these interaction behavior policies together with the motion prediction capability, the robotic agent can interact with human partners effectively.

In essence, we postulate that while modeling close-proximity human-human dyadic movement behaviors, how such models might facilitate similar fluent human-robot interactions has to be considered simultaneously. This overarching goal necessitates a bidirectional analysis and construction. A top-down investigation of dyadic interactions reveals the variety of possible cases, which, in turn, together with a bottom-up analysis, suggests the effective features that can be used to construct robot control policies for close-proximity HRI.

In this work, HHI, with a focus on shared workspace settings and repetitive tasks, is modeled as a graph-based representation. By incorporating insights from attention theory, psychology, and task generalization studies, this graph structure defines the possible dyadic interaction points for a task. In a next step, the edge combinations of the graph were generated and evaluated to identify possible representations. The high number of combinations in this initial graph model was analyzed in terms of their feasibility to form the full ontology representation (20 distinct cases). Then, a reduced ontology was obtained by the quantitative analysis of the HHI experiments conducted for the full model. Realistic test settings had to be designed that represent each ontology case. These test scenarios included a variability in task setup and dyad positioning in order to verify the robustness of the ontology. The design process as well as the data analysis enabled a methodical reduction procedure to decompose initial HHI representation into seven distinct ontology cases.

User studies that cover an extensive set of scenarios have been conducted and the movement data is analyzed thoroughly, which, in turn, supports our ontology abstraction. The data-driven analysis has shown that the reduced ontology cases can be classified with high accuracy. Additional classification analysis was conducted with data unknown to the classifier, which demonstrated the generalization capability of the proposed model in terms of human movement behavior. For a subset of the ontology cases, it has also been shown that the ontology representation is invariant to the dyad positioning. Lastly, a unique dyadic interaction behavior policy per ontology case has been learned by training long short-term memory (LSTM) networks, enabling the transfer of those controllers to collaborative robotic agents. The main contributions of our work can be summed up as follows:

- The proposed ontology provides a general, unified, and reusable framework as well as a baseline for the categorical analysis and classification of interactions.

- The ontology is supplemented with an imitation learning formulation to model human motion behaviors that enables predicting and imitating them during close proximity HRI.

The article is structured as follows: In Section 2, we review related work, followed by a model for HHI in Section 3. From the proposed model, we derive an ontology of HHI in Section 4. In Section 5, we introduce the imitation learning approach with LSTMs for human motion prediction and controlling a robot end-effector. The proposed ontology is validated with two experiments, as described in Section 6. The results of the experiments are presented in Section 7, and discussed in Section 8. Lastly, we draw a conclusion and give an outlook on future work in Section 9.

\section{RELATED WORK}

In this section, we briefly explain the related work both in the field of HRI and several relevant fields for $\mathrm{HHI}$ in a shared workspace, i.e., human motion prediction, as well as social psychology and neuroscience studies for interactions. 


\subsection{Human-Robot Interaction}

Here, we review recent studies on shared workspace interaction that are closer to the settings analyzed within our work. Learning human motion behavior from recordings is a common approach to model and then predict human motions in an HRI scenario. Hayne et al. (2016) presented a cost map approach, in which a voxel grid of costs is constructed. Costs are applied to each voxel, which has already been occupied by a human during a task. The robot can plan a collision-free path with the help of the cost map. The advantage of this approach is its simplicity; however, it cannot consider unobserved human behavior. This drawback severely restricts the effectiveness of robot motion planning. In contrast, Mainprice et al. $(2015,2016)$ frame the interaction behavior as an optimal control problem by assuming that the motions are optimally generated with respect to a set of cost functions. They used inverse reinforcement learning to learn such a cost function, followed by a stochastic motion planner for human motion prediction. They have shown the reliability of their approach for a few cases of HHI; since only one interaction scenario was tested, it is still unclear if this approach can be used for other scenarios. Furthermore, how those cost functions differ from case to case, and whether they can provide deeper insights on interaction features are still interesting open questions. In this work, we analyze multiple interaction scenarios in order to extract some base interaction cases, for which the aforementioned work could be utilized.

The same problem can be solved by considering the human as a dynamic obstacle, and use motion planning algorithms (Brock and Khatib 2002; Oguz et al. 2017; Park et al. 2013). In those studies, the focus is on planning motion behavior of the robot with respect to the changes in the environment for safety; hence, the resulting behavior is reactive. However, natural interaction does not only require safety and reactiveness but also a reciprocal responsive behavior. In our work, we focus on the analysis of the HHI scenarios to extract the features of such natural, safe, yet effective interaction behaviors. In that regard, the proposed ontology will also be useful for those motion planning frameworks.

Another type of dyadic interaction is physical human-robot joint actions, where a human-robot team works on the same object, e.g., carrying a table. The perception and control problem shifts from motion prediction and adaptation to force interactions, which are transmitted through the object. These force interactions have to be interpreted carefully to realize whether they indicate that more resistive force has to be applied (Rozo Castañeda et al. 2013), or whether it is a signal from the collaborator to switch directions or roles (Calinon et al. 2009; Donner and Buss 2016; Li et al. 2015; Oguz et al. 2012). In our work, non-physical dyadic motion behaviors in a shared workspace are investigated instead of joint physical actions. This necessitates identification of relevant features for interaction behavior control.

Joint actions and action coordination, where the partners share a common goal, have been the focus of numerous HRI studies. By understanding human movement behaviors during such scenarios, effective collaboration strategies have been developed for HRI settings (Hoffman 2013; Lenz et al. 2008; Mutlu et al. 2013; Shah et al. 2011). By combining subjective and objective evaluations, Hoffman (2007) proposes a number of team fluency metrics, which have been used in those studies. In this work, we focus on latent features related to the movement patterns of human partners, and evaluate their movement fluency and coordination only qualitatively. Nevertheless, the ontology proposed in this work can be extended to collaboration scenarios where the partners share a common goal, e.g., object handover tasks between a robot and a human collaborator, for which a synchronous behavior is necessary. The difficulty in this task is to predict the handover pose of the human, since the robot end-effector has to adapt to it as well as the intentions during the handover task. At the start, the human, as the receiver of the object, has to adapt to the pose of the robot (Edsinger and Kemp 2007). This is followed by the robot identifying and reacting to distinct 
handover poses (Cakmak et al. 2011). Finally, the social interaction between human and robot is optimized such that the intention of the robot to hand over an object becomes clear (Admoni et al. 2014; Strabala et al. 2013). We view handover tasks as a distinctive but still important case of HHI, which can be incorporated into our proposed ontology, as will be explained later in Section 3.1.

\subsection{Human Motion Modeling and Prediction}

Human motion behavior in an interaction scenario comprises two critical components. On the one hand, the mutual adaptation has to be considered, and on the other hand, the motion related to the specific task of the individual has to be considered. The latter will be reviewed here as the learning and adaptation phases of dyadic interaction are not considered in this study.

Affordance concept is an influential formulation to investigate motion behaviors, and it is mostly used to predict possible actions on an object, while abstracting its utility. Ugur et al. $(2015,2015)$ use a continuous object representation in order to compute the effects of an action on an object by computing the difference between the representations before and after the action. The proposed framework was able to reliably predict the effects of certain actions, and also to generate plans if the desired effect is given. In a similar work, Koppula et al. $(2013,2013,2014)$ used the affordance concept to predict human motion trajectories of possible actions, which are associated with an object. Affordances were encoded in an energy function that is to be maximized. The reliability of the approach was also demonstrated in predicting human motions within a short time horizon. Even though the feasibility of this approach has been claimed for interaction scenarios if an interaction cost is added to the energy function, it has yet to be shown. Our approach is quite similar to the concept of affordances. Based on the setup of the interaction, we estimate an interaction case that enables generating a feasible interaction trajectory as the prediction. However, the difference is that we are only interested in the interaction process rather than the objects related to it.

Similar to affordances, Semantic Event Chains (SECs) approach has been proposed by Aksoy et al. (2011, 2013). They encode tasks as a sequence of graphs, which represent the topology of the task at a given moment. This high-level abstraction enables grouping of similar objects and tasks, such that task learning can be simplified. In this regard, a framework has been proposed in which SECs can be used as a form of manipulation library in order for a robot to execute different tasks (Aein et al. 2013). Similarly, Wörgötter et al. (2013) abstracted actions and generalized manipulations according to their topological effects, instead of the objects. They examined the contact between objects during manipulations and derived a small subset of contact situations, which reliably describe almost all possible singlehand manipulations. In this work, we make use of their manipulation abstraction, in order to make our interaction model task invariant.

Related to our imitation learning approach with LSTM units, Murata et al. (2018) proposes learning interaction behaviors with RNNs, and extracting HRI information from image data. Using only HRI data, rather than HHI observations, joint position controllers are learned successfully. The focus of the study is on discrete action selection and adaptation depending on the human partner's action, and thus interfering motion trajectories were not considered. Similarly, high-level action sequences rather than control of the movement has been investigated by advanced LSTM architectures (Nguyen et al. 2017). RNN-based policies have also been used recently on modeling pedestrian movement behavior (Alahi et al. 2016; Jain et al. 2016; Pfeiffer et al. 2017; Vemula et al. 2017), but the extendibility of those approaches to robotic agents, especially to manipulators, has not been investigated.

\subsection{Psychology and Neuroscience}

Once the task-related motion prediction is taken into account, the perception aspect of interaction has to be considered as well. Therefore, it is reasonable to leverage insights from psychology and 
neuroscience as they investigate the factors involved in interaction. Among a multitude of topics, entrainment, or synchronization (Lorenz et al. 2014; Sebanz and Knoblich 2009) is arguably one of the broadly studied interaction components. Various studies show that entrainment emerges, even if humans are not instructed to synchronize their motions for dyadic tasks (Sebanz and Knoblich 2009; Watanabe 2008).

Another important set of properties for $\mathrm{HHI}$ is related to the theories on attention. During $\mathrm{HHI}$, humans have to split their attention between their task and the interacting partner, resulting in an attention distribution. Corbetta et al. (2002) explain those mechanisms and show that attention is drawn to the biggest stimuli. However, this trend changes during task executions. Prior information about the task helps the attention system to filter out unimportant stimuli, while paying special attention to task relevant stimuli (Desimone and Duncan 1995; Egeth and Yantis 1997; Folk et al. 1992). For HHI in a shared workspace, the dominant percept is vision and the dominant stimulus are fast motions that may lead to collisions (Gao et al. 2008; Itti and Koch 2001). For our study, these behavioral properties on synchronicity and attention distribution are also considered for constructing our ontology and interaction models.

This review has shown relevant work in the field of HRI, followed by the two components of $\mathrm{HHI}$ in a shared workspace, task related motions and effects, as well as relevant work on human interaction behavior. In the next section, these components are merged into a model for dyadic interactions.

\section{MODELING INTERACTIONS FOR HHI}

For a systematic analysis of $\mathrm{HHI}$ in a shared workspace scenario, it is first necessary to determine the components that influence those interactions. Sebanz et al. (2009) identifies three key questions that describe these influences: "What happens? Where does it happen? And, when?" These questions are linked to the inference of actions and their goals (what), the locations at which the motion or action takes place (where), and the timing, in other words, synchronization of actions (when). In this section, we use these three questions as a guideline to create an interaction model for HHI. We first explain our insights into the posed questions, followed by a derivation of an interaction model. In order to help with following our explanation, we use the interaction case shown in Figure 1 as a recurring example. This example consists of a pick and place task, combined with a setup that forces the dyad to cross arms during the execution.

What. The question of "what happens" can be answered from two different levels of abstraction. The higher level of abstraction is the intention of the interacting agents, i.e., what they want to do with the task related object(s). There have been several approaches on how to predict human intention, such as affordances (Koppula and Saxena 2014; Ugur et al. 2015), or library-based methods (Aein et al. 2013). In our formulation, we assume that the intended task of the collaborator is either already known or can be correctly inferred at the start of interaction. This is a reasonable assumption, especially for repetitive close proximity interaction tasks such as collaborative manufacturing scenarios. Once task intention is presumed, the next level of abstraction, i.e., how the actors are going to realize their intention by their motion, becomes relevant. Thus, in a next step, the task is split into relevant subtasks, resulting in a sequence of actions. For the given pick and place example, this is the sequence of picking up an object, bringing it to the target position, and then placing it. The partners then use this information to estimate the influence of each other's task sequence on their task. This sequential aspect can be further abstracted if the subtasks are generalized as it has been done in (Wörgötter et al. 2013). This generalization allows to treat individual small motions as black boxes: Using the pick and place example, whether the object is picked up or placed down at one of the predetermined locations is insignificant. It just has to be 
known that a task-related event takes place at that location. Since less information about the actual manipulation action needs to be processed, the attention on the interaction, i.e., the relevant task sequences that influence each other's tasks, becomes critical. This allocation of attention directly leads to the question of where the important interaction sequences happen.

Where. The question of "where does it happen" is again analyzed by two levels of abstraction. The first is the allocation of the shared workspace. Given our pick-and-place example, this is described by which person grasps over the other's arm, i.e., the rough location each interacting agent can occupy during their next transport motion. In addition to the allocation of space, joint attention is also relevant to the allocation of tasks (Sebanz and Knoblich 2009). For close proximity and repetitive interactions such as collaborative manufacturing scenarios, we assume that the tasks are pre-allocated. The joint attention is mostly on the distribution of space. During preliminary experiments, we observed that this allocation is relatively stable after a short period of adjustment in the early phases of interaction. The same can be assumed for renegotiation after a stable allocation is discarded. Hence, we assume that space allocation is negotiated for a short period and stays mostly the same thereafter. Consequently, attention needs to be paid only to interaction related stimuli (Corbetta and Shulman 2002; Desimone and Duncan 1995), i.e., task relevant motions. Discordant movement behaviors, e.g., those that are faster than expected or that might lead to collision, increase the partners' attention to the motion. The same holds true for negotiation phases. In essence, timing is tightly coupled to these conditions, and thus, plays a crucial role. Taking our example case (Figure 1), if both actors move into the workspace at the same time, a collision may occur, and more attention has to be paid on the partner's movement. However, if they move into the shared workspace alternately, then clearly the dyad does not obstruct their motion reciprocally.

When. Considering the arguments on what happens and where, we can argue that, in general, attention is increased toward motions only if they are timed such that they can result in a collision. If the confined workspace is restrictive for joint occupation, it is a reasonable strategy to time the motions such that the space is shared in turns. This approach aligns with the concept of entrainment or synchronization, which expresses the tendency of humans to fully synchronize their motions (Lorenz et al. 2014). Although asynchronous motions within the shared space reduce the mental effort through attention, this strategy may no longer be valid if time efficiency is critical, as in most manufacturing scenarios. Especially, if one partner's task takes longer, then it becomes infeasible for the other to wait. Taking our pick-and-place example, this would be the case if assembling the brick requires a more complex action such as screwing rather than just plugging in. In essence, for an interaction involving individual tasks, timing might not be a key determinant to define the interaction but more of a variable of it, which can be treated as a control signal. Most interactions still work with bad timing, while well-timed actions improve the interaction.

\subsection{Interaction Model}

In this section, based on the insights given for the posed questions, we propose an interaction model with three assumptions. The model is constrained to a shared and spatially constricted workspace $(A 1)$. Furthermore, we assume that the interaction is situated in a structured environment, such as a manufacturing setup (A2). This second assumption includes that tasks are known and distributed beforehand, and that tasks are executed repeatedly and at least to a degree time efficiently. The repetition of the tasks ensures that the workspace is usually allocated, and that we can observe an interaction pattern over time, while still capturing the variance of similar motions. Movement patterns are determined w.r.t. the synchronization of the motions, and ensure comparable interaction behaviors. Based on these assumptions, we formulate the task sequence for 


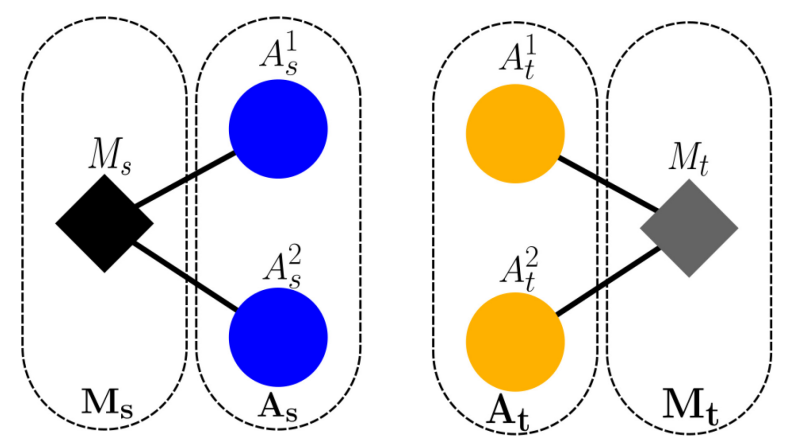

Fig. 2. Graphical representation of the proposed interaction model. Action $A_{a}$ (round) and motion $\mathbf{M}_{\mathrm{a}}$ (squared) spaces are colored separately. Each partite vertex set is framed.

follow-up task executions as a sequence of approaching and withdrawing motions, and an action phase in between, similar to Wörgötter et al. (2013). With the insights from attention theory, we can merge the withdraw and approach phases into a motion phase, since it does not matter what motion type is performed as long as it possibly interferes with another motion. In other words, we define an action as a movement with limited spatial variance, e.g., picking up an item, whereas a motion has a bigger spatial coverage, e.g., a point-to-point reaching motion. This reduces the task procedure to a sequence of actions and motions.

Lastly, we assume that a task is defined by two distinct locations, in which actions take place $(A 3)$. For a structured analysis, this restriction is imposed on both agents, i.e., the pick-up and placement locations are spatially different in our example pick-and-place task. Furthermore, alongside the reasoning of Wörgötter (2013), we model interactions such that the model is task independent, e.g., for a crossing arms movement behavior, the crucial part is the transport motion, and whether it is a pick-and-place task or fixing a stripped screw is irrelevant for the model structure. Therefore, we assume that the micro motions of each action, i.e. placing the brick or screwing, take place in a black-box, which we call action spaces. Note that these micro motions are restricted spatially around the manipulated object and, thus, most likely to be ignored by the partner's attention system. These action spaces are connected to each other by a motion space, for which the trajectory in between both action spaces is abstracted, i.e. the shape of the trajectory is not taken into account. This allows including the variance of individual behaviors, as well as the motion adjustments due to environmental influences, e.g., obstacle avoidance, into our model.

Using the above reasoning, we create a graph-based model of interactions. Two fully connected bipartite graphs $K(2,1)$ are combined into a 4-partite graph with the following vertices and edges:

$$
\begin{gathered}
\mathrm{G}=\left\{\mathbf{A}_{\mathrm{a}}, \mathbf{M}_{\mathrm{a}}, \mathbf{E}\right\}, \\
\mathbf{A}_{\mathrm{a}}=\left\{A_{a}^{1}, A_{a}^{2}\right\}, \quad \mathbf{M}_{\mathbf{a}}=\left\{M_{a}\right\}, \\
\mathbf{E}=\left\{\left\{A_{a}^{1} M_{a}\right\},\left\{A_{a}^{2} M_{a}\right\}\right\}, a \in\{\mathrm{s}, \mathrm{t}\}
\end{gathered}
$$

Here, $G$ represents the interaction model for two actors, $\mathbf{A}_{\mathbf{a}}, \mathbf{M}_{\mathrm{a}}$ the disjoint sets of vertices that represent the model, and $\mathrm{E}$ the edges connecting them, where $s$ and $t$ refer to self and the other, respectively. The vertices of the set $\mathbf{A}_{\mathbf{a}}$ are called action (manipulation) spaces and represent the blackbox in which actions take place, such as picking up or placing. The vertex of the set $\mathbf{M}_{\mathrm{a}}$ represents the motion trajectory connecting the two action spaces. Hence, a sequence of tasks can be represented by a sequence of vertices of a graph. Furthermore, this graph contains all locations at which an interaction can take place, be it motion or action spaces. A graphical representation of this model can be found in Figure 2. Corresponding to our example of a pick-and-place task, the 
picking up of the object would be represented by $A_{a}^{1}$, while the placement of the object by $A_{a}^{2}$, and the transport motion by $M_{a}$. Based on this model, we will derive an ontology for HHI in the next section by exploring all possible edge combinations of the interaction model.

\section{HUMAN-HUMAN INTERACTION ONTOLOGY}

The proposed 4-partite interaction model provides us with a tool to systematically analyze HHI. In a first step, all possible edge combinations for the interaction model are generated and analyzed. For this analysis, an interpretation of the individual edges with respect to human interactions is essential to reduce the number of feasible cases. Each edge has to represent a specific interaction between the dyad members during the execution of an arbitrary task. Three types of interaction are specified as follows:

$-E_{1}(k, l)=\left\{A_{s}^{k} A_{t}^{l}\right\} \forall k, l \in\{1,2\}:$ An interaction between the action spaces of the dyad. We interpret this as a shared action space. Examples for such an interaction would be a handover task or taking objects from the same stash.

$-E_{2}(k, a)=\left\{A_{a}^{k} M_{\bar{a}}\right\} \forall k \in\{1,2\}$ and $\forall a \in\{s, t\}:$ An interaction between an action space of one agent and the trajectory of the other; here, the opposite agent is denoted by $\bar{a}$. We interpret this as one actor grasping over the action space of the other. As a result, the respective agent has to avoid the other's action space for improved cooperation.

$-E_{3}=\left\{M_{s} M_{t}\right\}:$ An interaction between the trajectories of the agent itself $(s)$ and the other agent $(t)$. This can be interpreted as crossover motion, where agents' trajectories intersect. Therefore, the agents have to avoid collision with their partners (Figure 1).

The combination of the edges' results, i.e., three possible edges per node, makes a total of nine possible edges, resulting in a total number of $N=2+\sum_{p=1}^{8} \frac{9 !}{p !(9-p) !}=513$ interaction scenarios. The total number $N$ is computed by either successively adding edges to the unconnected graph, or by fully connecting all vertices, and then eliminating individual edges. In order to reveal the reasonable cases, the following simplifications and reductions are applied in the form of edge equivalences:

-First, all edges violating model assumption $A 3$ (see Section 3.1) are eliminated. All graphs containing the edge set $E=\left\{E_{1}(1, l), E_{1}(2, l)\right\} \vee\left\{E_{1}(k, 1), E_{1}(k, 2)\right\}$ violate this assumption and are therefore omitted. According to the edge interpretation, two action spaces from the same partition are merged, and thus eliminate the necessity of a motion.

-A similar problem arises, when two action spaces are merged, i.e., when an edge $E_{1}$ exists. If there is an additional incident edge $E_{2}$, an agent would grasp over their own action space. This can, as well, be seen as part of the grasping process, and thus merged into the action space. Therefore, all graphs containing the edge set $E=\left\{E_{1}(k, l), E_{2}(k, s)\right\} \vee$ $\left\{E_{1}(k, l), E_{2}(l, t)\right\}$ are omitted.

- Besides edge reductions due to the model definition, symmetry can also be used to reduce the number of cases. Since initial and final points are insignificant for the model, and the positions of the action spaces can be set arbitrarily as long as they fulfill the edge interpretations, symmetry of an agent's action spaces can be assumed. Therefore, based on the assumption that $A_{s}^{1} \simeq A_{s}^{2}$ and $A_{t}^{1} \simeq A_{t}^{2}$, a graph $\mathrm{G}=\left\{\mathbf{A}, \mathbf{M}, \mathbf{E}_{\mathbf{x}}, E_{2}(1, a)\right\}$ is isomorphic to a graph $\mathbf{H}=\left\{\mathbf{A}, \mathbf{M}, \mathbf{E}_{\mathbf{x}}, E_{2}(2, a)\right\}$ if they share the other set of arbitrary edges $\mathbf{E}_{\mathbf{x}}$. As a result, $\mathrm{G} \simeq \mathrm{H}$ holds.

- Since the relationship between dyad motions is investigated, models with symmetry in motion spaces can be discarded, i.e., whether agent $i$ or agent $j$ grasps over the other's action 

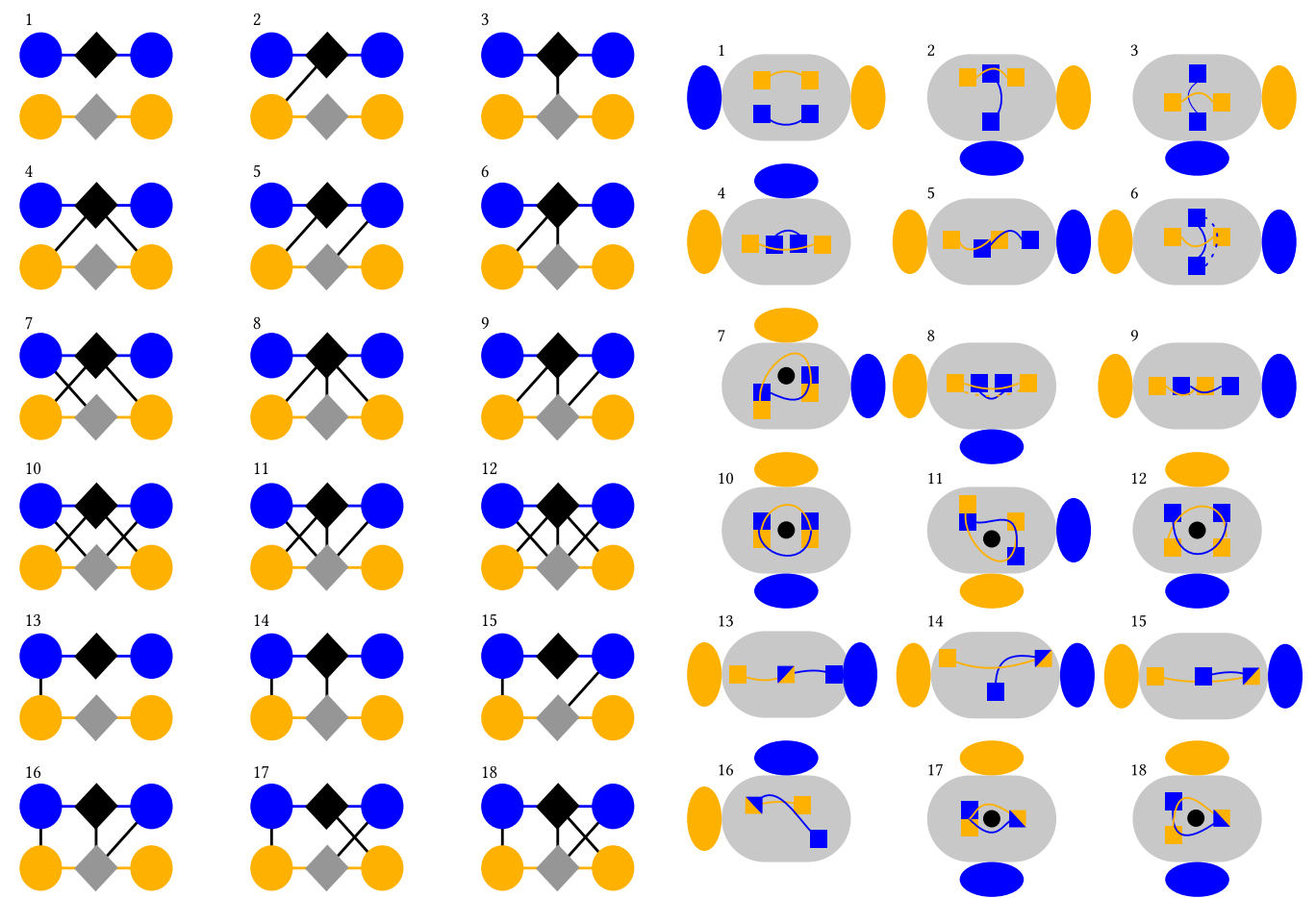

$14+15$
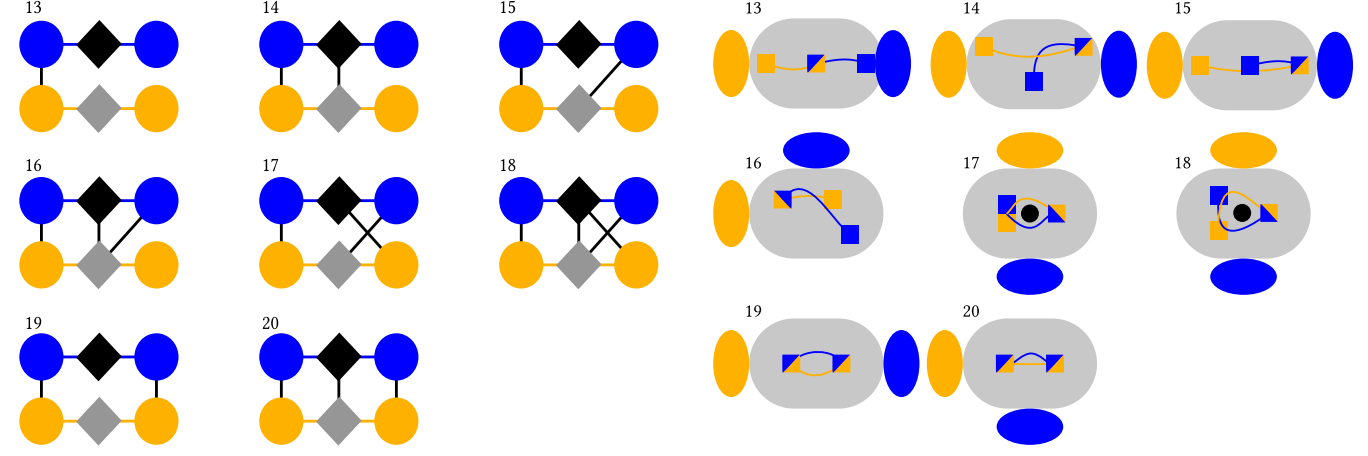

(a)

(b)

Fig. 3. (a) Graph representation, and (b) exemplary setups for each case in the full ontology. (a) Action (round) and motion (squared) spaces are colored separately. (b) Gray area depicts the shared workspace, while the black circle represents an obstacle blocking the path and dashed lines show the motion of the elbow, where necessary.

space is not critical. In combination with the previous assumption, $E_{2}(k, s) \simeq E_{2}(l, t)$ holds true for any indices $k, l$.

Based on these simplifications, and symmetry assumptions, the number of feasible cases could be reduced to 20 interaction cases (Figure 3(a)), which represent the full ontology. Exemplary setups for each of these 20 interaction cases are illustrated in Figure 3(b). Obstacles are introduced for some cases that enforce interaction variations in order to extend the analyzed dyadic movement behavior set. If those obstacles are removed, the interaction would change (as can be seen for case 18 , which becomes very similar to case 16 , if the object is removed), since the blue agent is no longer forced to grasp over one of the yellow action spaces.

\subsection{Ontology Reduction}

As it can be seen in the examples, some interaction cases of the full ontology look similar. Based on the theoretical and heuristic deductions as well as an experimental verification, the full ontology of 20 cases could be reduced to seven cases, which are called the reduced ontology for the rest of the 
article. For the theoretical deduction, the degree-of-freedom of action and manipulation spaces are observed. An action or manipulation space is assumed to have a degree-of-freedom if the corresponding vertex has no edges from the set $E=\left\{E_{1}, E_{2}, E_{3}\right\}$. For the reduction, the following cases were merged, and a proto-case is highlighted in bold for each reduced case:

-Zero degree-of-freedom cases: 10,12, 17, and 18. These are the ontology cases that are constrained the most. Due to the high number of $E_{2}$ interactions, the motions of each agent are thoroughly restricted. Both agents have to grasp over two action spaces, or have a shared action space, which limits the possible trajectories to the one seen in the examples in Figure 3(b). Such setups are necessary to enforce $E_{2}$ interactions. Due to the constraints of those setups and the elbow kinematics of a human, there is barely any motion redundancy left, making these cases some of the easiest to predict. Furthermore, the kinematic restriction of the elbow on one side of the workspace enforces a strict timing between agents to work efficiently. Consequently, this case restricts the motion of the agents the most due to its high number of action space interactions. This stands in contrast to cases 19 and 20, which are are also restricted in their action spaces but have less restricted trajectories due to the absence of $E_{2}$ interactions.

- One degree-of-freedom cases: 7, 11. These cases are similar to case $\mathbf{1 0}$. The only difference is that one agent has more options for one of their action spaces. This results in a relaxed trajectory planning for one agent and, thus, a broader range of interactions. This can also be seen in the possible variability of the experimental setup of these cases.

- Two degree-of-freedom cases: 5, 6, 9, 13, and 19. These cases have a similar spatial configuration to the extent that either one of the action spaces of an agent is in close proximity to one of the other agent's, or that they share an action space. For cases 5 and 9 , the close proximity of two action spaces is necessary in order to ensure the two action and motion space interactions. Additionally, the merged cases require asynchronous motions. For cases 13 and 19, this is necessary due to the shared action space, which limits the space for executing the action and, as such, should be used in sequence. For cases 5 and 9, an in-line placement of the action spaces is required to realize these scenarios if no obstacles are introduced (Figure 3(b)). Otherwise, they will be similar to cases 7 and 11. This in-line placement leads to one person partially obstructing the other's action space. In essence, asynchronous motions are favorable, which is similar to shared workspace cases such as case 2 . Lastly, there is a second shared workspace in case 19; however, it does not impose any additional restrictions, since asynchronous behavior is already a favorable option.

- Two degree-of-freedom cases: 4, 8, 14, 15, 16, and 20. These cases are similar due to their spatial configuration. All of them have one person grasping over both of the other's action spaces, while having no interaction on their own action spaces. Their dissimilarity lies in the crossing of motion spaces in case 8 . However, since the motion space has to lie in between the action spaces, there will be no influence of this interaction possibility on the overall interaction. The first person always grasps over both action spaces; therefore, the inclusion of an additional motion space does not change the overall behavior, while the second person is mostly working in the other's motion space, which stays the same in case 4 and 8. Considering case 15, one of the action spaces is merged, making it a shared workspace equivalent of case 4 . This merged space also allows for a relaxation of assumption A3: Since one agent is grasping over the other's action spaces, it could also be assumed that the other is executing a stationary task in one action space. The overall interaction would be roughly the same. This possible relaxation of $A 3$ further shows the flexibility of the proposed ontology. 


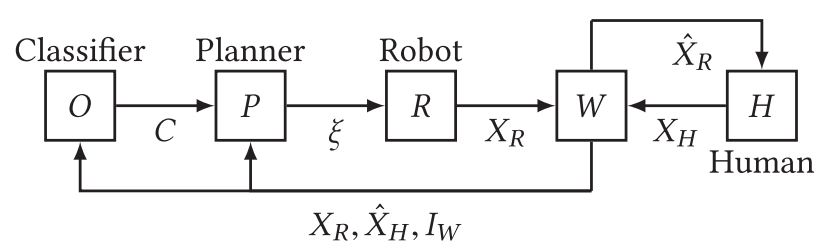

Fig. 4. Control schematic for using the ontology in HRI scenarios. Human $H$ and robot $R$ are interacting in a shared workspace $W$. Humans adapt their interaction based on their proprioreception and the observed robot motion $\hat{X}_{R}$. The robot adapts to the human agent in two feedback cycles using observations of the human $\hat{X}_{H}$, its own feedback $X_{R}$, and the workspace composition $I_{W}$. The inner loop motion planner $P$ reacts to human motions based on the current ontology case $C$, while the outer loop ontology classifier $O$ estimates the current case based on the feedback data.

\subsection{Ontology Application}

Representing close-proximity interactions by graph structures allows us to investigate dyadic movements within various settings from a top-down perspective. The subsequent data-driven and bottom-up analysis of the resulting ontology informs us about the similarities and differences between cases. However, utilizing such an ontology for similar HRI scenarios requires further considerations. In essence, it needs to be embedded into a broader control scheme, such as shown in Figure 4. For simplicity, we omitted the high-level controller that determines the target objective of the robot. In this example, the ontology is split into an ontology classifier $O$ that determines the current ontology case based on human $\hat{X}_{H}$ and robot $X_{R}$ trajectories as well as general information about the workspace $I_{W}$, and a trajectory planner $P$ that realizes human-like behavior for the robot $\xi$, i.e., encodes human behavior. This reference is then used by the actual robot $R$ to interact with the human agent $H$ in a shared workspace $W$. An example of such a motion planner is described in the following section, and an exemplary classifier is demonstrated in Section 7. This simple example can be updated based on the sensor capabilities and goals. It might be a good idea to bootstrap the ontology classifier with a prior knowledge of the workspace. The classifier could further be improved by also detecting the current state of human and robot in the graph structure $\left(A_{a}^{i}, M_{a}\right)$ and giving that information to the planner to enhance the HRI. In the following, we present a simple motion planner in the form of an LSTM network that only depends on the ontology case.

\section{HUMAN INTERACTION BEHAVIOR MODELING}

In this section, the learning framework we used in this work is briefly introduced. The main idea is to model human interaction behavior control with recurrent neural networks (RNNs) by learning from human-human interaction movement data. This data is available in the form of a time parametrized trajectory $\xi$ in a configuration space, which can be defined as follows:

$$
\xi=\left[q_{1} \ldots q_{N}\right]
$$

where $q_{i}$ are the vectors of configuration, and $N$ denotes the number of waypoints. RNN models are well-suited to learn from such sequential data. Like feedforward networks, RNNs are typically organized in layers, each representing a distinct timestep. Input signals are propagated through the network starting with the network inputs to the first layer. For the modeling in this work, we specifically use LSTM cells in the RNN structure. Let $\mathbf{x}_{t}$ be inputs and $\mathbf{y}_{t}$ be outputs of a layer $j$ at timestep $t$. Furthermore, let $\odot$ denote the element-wise multiplication for two vectors, and $\sigma$ as well as $\phi$ denote different nonlinear activation functions. Then, the LSTM network comprises the 
following equations:

$$
\begin{aligned}
& \mathbf{i}_{t}^{j}= \sigma\left(\mathbf{W}_{i, x}^{j} \mathbf{x}_{t}^{j}+\mathbf{W}_{i, y}^{j} \mathbf{y}_{t-1}^{j}+\mathbf{b}_{i}^{j}\right) \\
& \mathbf{f}_{t}^{j}= \sigma\left(\mathbf{W}_{f, x}^{j} \mathbf{x}_{t}^{j}+\mathbf{W}_{f, y}^{j} \mathbf{y}_{t-1}^{j}+\mathbf{b}_{f}^{j}\right) \\
& \mathbf{o}_{t}^{j}= \sigma\left(\mathbf{W}_{o, x}^{j} \mathbf{x}_{t}^{j}+\mathbf{W}_{o, y}^{j} \mathbf{y}_{t-1}^{j}+\mathbf{b}_{o}^{j}\right) \\
& \mathbf{g}_{t}^{j}= \phi\left(\mathbf{W}_{g, x}^{j} \mathbf{x}_{t}^{j}+\mathbf{W}_{g, y}^{j} \mathbf{y}_{t-1}^{j}+\mathbf{b}_{g}^{j}\right) \\
& \mathbf{c}_{t}^{j}=\mathbf{f}_{t}^{j} \odot \mathbf{c}_{t-1}^{j}+\mathbf{i}_{t}^{j} \odot \mathbf{g}_{t}^{j} \\
& \mathbf{y}_{t}^{j}=\mathbf{o}_{t}^{j} \odot \phi\left(\mathbf{c}_{t}^{j}\right)
\end{aligned}
$$

These characterize each layer of a multilayer LSTM network structure, and a final linear output layer takes the form $\mathbf{y}_{\text {out }}=\mathbf{W}_{\text {out }} \mathbf{x}_{\text {out }}+\mathbf{b}_{\text {out }}$.

\subsection{Learning with LSTMs}

Here, we explain how LSTM-based policies are used for our imitation learning approach. LSTM networks are trained on acceleration output sequences of the form $\mathbf{Y}_{k}=\left[\mathbf{y}_{0, k}, \mathbf{y}_{1, k}, \ldots \mathbf{y}_{n_{k}-1, k}\right]$ and spatial input sequences such as $\mathbf{X}_{k}=\left[\mathbf{x}_{0, k}, \mathbf{x}_{1, k}, \ldots \mathbf{x}_{n_{k}-1, k}\right]$, with $k$ being the batch index. Using optimization and backpropagation, the minimization problem

$$
\min _{\theta} \mathrm{D}=\frac{\sum_{k \in K}\left\|\mathrm{Y}_{k}-\hat{\mathbf{Y}}_{k}\right\|_{2}^{2}}{\sum_{k \in K} n_{k}},
$$

where $\theta$ represents policy parameters, i.e., the weights of the LSTM network (and $\hat{\mathbf{Y}}_{k}$ is the estimated acceleration output sequence) for a given spatial input is solved.

The available data from human-human collaboration experiments (Section 6) is used to obtain $\mathrm{X}_{k}$ and $\mathrm{Y}_{k}$ by extracting features based on hand positions, $\mathrm{s}_{\mathrm{H}, \mathrm{P} 1}$ and $\mathrm{s}_{\mathrm{H}, \mathrm{P} 2}$, of the persons $\mathrm{P} 1$ and $\mathrm{P} 2$ in the collaboration experiment, respectively. It is assumed that the robotic agent is supposed to learn the movement control of P1 as part of the interaction scenarios. In order to eliminate a directional bias, we augment the original data by rotating it with $90^{\circ}, 180^{\circ}$, and $270^{\circ}$ (Amaral et al. 2014).

Input features are $(i) \mathfrak{f}_{\mathrm{H} 2 \mathrm{G}, t}$, storing the distance between P1's current hand and goal position, $\mathbf{s}_{\mathrm{H}, \mathrm{P} 1, t}$ and $\mathbf{s}_{g, \mathrm{P} 1, t}$, respectively, and $(i i) \tilde{f}_{\mathrm{H} 2 \mathrm{H}, t}$, describing the relation between two persons' hands, $s_{\mathrm{H}, \mathrm{P} 1}$ and $s_{\mathrm{H}, \mathrm{P} 2}$, and is computed by a slightly modified formula of the potential fields approach as presented in Oguz et al. (2018a). The output feature is the end-effector acceleration (P1's hand) given by

$$
\mathbf{a}_{H, \mathrm{P} 1}=\nabla^{2} \mathbf{s}_{\mathrm{H}, \mathrm{P} 1}
$$

This finally gives the following structure to the input and output sequence data fed to the LSTM model

$$
\begin{aligned}
\mathbf{X}_{k} & =\left[\begin{array}{llll}
\mathfrak{f}_{\mathrm{H} 2 \mathrm{G}, 0}^{k} & \mathfrak{f}_{\mathrm{H} 2 \mathrm{G}, 1}^{k} & \ldots & \mathfrak{f}_{\mathrm{H} 2 \mathrm{G}, n_{k}-1}^{k} \\
\mathfrak{f}_{\mathrm{H} 2 \mathrm{H}, 0}^{k} & \mathfrak{f}_{\mathrm{H} 2 \mathrm{H}, 1}^{k} & \cdots & \mathfrak{f}_{\mathrm{H} 2 \mathrm{H}, n_{k}-1}^{k}
\end{array}\right] \in \mathbb{R}^{6 \times n_{k}} \\
\mathbf{Y}_{k} & =\left[\begin{array}{llll}
\mathbf{a}_{H, \mathrm{P} 1,0} & \mathbf{a}_{H, \mathrm{P} 1,1} & \ldots & \mathbf{a}_{H, \mathrm{P} 1, n_{k}-1}
\end{array}\right] \in \mathbb{R}^{3 \times n_{k}}
\end{aligned}
$$

In addition to the mean squared error (MSE) output layer described above, we also implement a Gaussian mixture model (GMM) output layer to provide a stochastic alternative for the proposed 
policies. This allows to extend our analysis to consider uncertainties within the proposed interaction scenarios. Parameters of a GMM that best represent the data is learned. It is characterized by the following loss function

$$
E(z)=-\log \left(\sum_{k=1}^{K} \frac{\phi_{k} \exp \left(-0.5\left(z-\mu_{k}\right)^{T} \Sigma^{-1}\left(z-\mu_{k}\right)\right)}{\sqrt{2 \pi\left|\Sigma_{k}\right|}}\right),
$$

where $K$ represents the respective mixture component, and $\phi_{k}, \mu_{k}, \Sigma_{k}$ are the mixture probability, mean, and covariance for each component. The negative log likelihood $E$ of samples $z$ is minimized such that, iteratively, parameters $\phi_{k}, \mu_{k}, \Sigma_{k}$ are updated and converged to an output mixture model that represents training data. During test time, control signals are sampled online from this output layer to generate interaction movements.

\section{HUMAN-HUMAN COLLABORATION EXPERIMENTS}

In this section, the experimental setup used to verify our ontology representation is explained. We conducted two separate experiments for verification. The first experiment used the ontology cases with the most degrees of freedom of motion in order to verify whether the ontology is feasible and also invariant to the relative positioning of the dyad. The second experiment was designed to test the rest of the ontology cases, while relaxing some of the controlled parameters of the first experiment. In this section, we first introduce the task selection for both experiments, followed by an explanation of the positioning selection of the participants. Lastly, we describe both experiments.

\subsection{Task and Collaboration Case Selection}

The goal of both experiments was to analyze interaction processes between humans sharing a confined workspace, and therefore, an adequate task has to be chosen. The task should be simple, while at the same time generalizable. The simplicity of the task ensures that the main focus of participants lies on the interaction instead of the task itself. The generalization capacity of the task extends the application of the results to a broad range of use cases. As a result of those considerations, we chose a pick-and-place task due to the fact that it is a routine activity and also part of almost all manufacturing tasks (Wörgötter et al. 2013). With this task selection, the collaboration focus is only restricted to the interaction trajectories.

\subsection{Relative Positioning}

Once the task is set, the next step is to define the relative positioning of the participants. In most interaction experiments, the participants are placed opposite to each other such that each participant can fully view the other participant's motion. However, in real-life scenarios, this may not always be the most feasible setup. An exploration of viable relative positions is necessary in order to verify its presumed influence on the interaction.

Positioning the interacting partners opposite to each other ensures that each of them are in the central field of view of the other. Hence, visual distinction of motions and objects is maximized, which, in turn, improves the reaction times. However, this capability of the human declines with each degree of eccentricity in the human field of view (Sardegna et al. 2002; Tynan and Sekuler 1982). For studying the effects of this change on human interaction behavior, we decided to evaluate three different sections of the field of view for the experiments: (i) central, (ii) peripheral, and (iii) extreme peripheral field of view. In the central field of view, the recognition of objects and motions is at its best, whereas the effectiveness of recognition declines in the peripheral field and vanishes in the extreme peripheral region (Sardegna et al. 2002). Unlike object recognition, motion directions can still be recognized even in the extreme peripheral vision, which is argued 


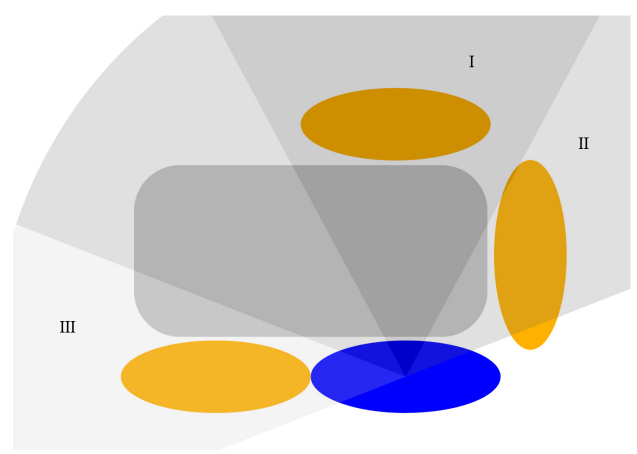

Fig. 5. The figure shows the analyzed relative dyad positioning: frontal (I), orthogonal (II), same side (III). The circular sectors represent the respective sections of the field of view.

to be due to the threat detection instincts (To et al. 2011). In order to test this hypothesis within our experiment, we decided to position participants in three different configurations (Figure 5).

\subsection{First Experiment}

The first experiment was designed to test two hypotheses:

$-H 1$ : Ontology cases are distinguishable with respect to the features extracted.

$-H 2$ : Ontology cases are invariant, in terms of the features, to the relative positioning of the dyad.

We used the ontology cases with the most degrees of freedom in setups, which are cases 1, 2, and 3 . These cases can be arranged for any relative positioning, since they impose no constraints on the relative positions of the action spaces with respect to each other. The positioning invariance was tested for the relative positions I, II, and III (Figure 5), resulting in a total of nine different setups for the experiment. The initial and final positions of each task were fixed in order to ensure that a different placement does not skew the results. One experimenter was also designated as one of the dyad for all the trials for providing steady and fluid behavior. This ensured comparable and consistent interaction behaviors by the subjects as well as the possibility to interrupt synchronous behavior-intentionally by the experimenter, if required-that broadens the spectrum of the timing behavior.

The experiment was conducted as follows: Experimental procedure as well as the task of the participant were explained to the subject, and the participant signed a form of consent. Next, the tracking markers were fit onto their body. At the start, a habituation phase of one minute was provided, where the experimenter and the participant executed their tasks for random setups, and the experimenter forced some interactions in order to familiarize the participant with the task as well as the experimenter. Afterward, the experiment was conducted in three sections, with three interaction cases each. Each section contained the three selected ontology cases from a different relative position. These sections were randomly selected. The cases in the section were arranged such that the free motion case 1 was always selected first, followed by a random order of cases 2 and 3. Free motion case (case 1) was set first as it does not enforce any interaction explicitly, and thus helps participants to get familiar with each relative position. Each of the interactions took place for exactly 1 minute, in which the participant and the experimenter should move Lego bricks that formed a tower-like structure from one position to another and vice versa as often as possible. An effective movement speed was encouraged in order to guarantee interactions and to avoid waiting of participants for each other. Between each interaction case, there was a short break 
of half a minute. The experiment has been conducted with 10 healthy participants, of which nine were male and one was female of ages $25.3 \pm 2.5$.

\subsection{Second Experiment}

In the second experiment, we tested the rest of the missing ontology cases, while relaxing the fixed setup constraint, i.e., the action spaces were slightly variated. Position invariance was not investigated as it is verified with the first experiment (Section 7) and also some of the more complex cases are only viable for a single setup. We used the relative positions I and II, and only tested the first hypothesis (H1) on classifying ontology cases between 4 and 20 in this secondary experiment. Those interaction cases were split into two groups, which were tested with a break in between. The first group consisted of cases without shared action spaces, i.e., cases 4-12, while the second consisted of the remaining cases, i.e., cases 13-20. The order of the cases within the group was randomized for each participant, while the order of the groups was kept fixed. This was preferred since the shared manipulation cases require some degree of synchronization, which gets easier once the participant is more familiar with the task and the partner. The second participant was again an experimenter in order to ensure a consistent natural interaction. The setups for each recording can be seen in Figure 3(b). The procedure of the experiment was similar to the first experiment. This second set of experiments has been conducted with eight healthy participants, of which seven were male and one was female of ages $25 \pm 2.4$.

\subsection{Recording and Preprocessing}

The recording was done by using the optical Qualisys tracking system with eight cameras. The participant and the experimenter were equipped with optical trackers positioned at the thorax, back, shoulder, elbow, wrist and hand. It was attempted to place the markers at the following anatomical landmarks: one at the xiphoid process, one at the Sternum, two randomly along the vertebra, two at the front and back of the glenohumeral rotation center, one at the elbow, one at the radial and ulnar styloid each, and one at the back of the hand. In the second experiment, two trackers were attached to the elbow for improving the tracking accuracy. Data was first labeled and corrected of erroneous trackings, before being exported to a Matlab file. Further processing of the data has been conducted in Matlab.

The data was recorded with a frequency of $250 \mathrm{HZ}(\sim 15,000$ frames/recording). In a first step, the recording was segmented automatically by detecting points at which the absolute velocity of the wrist was close to zero. Resulting segmentation errors were corrected manually. Subsequently, a 14-DoF arm model was used to reconstruct the joint angles of the model. Missing data points were interpolated linearly, and the resulting angles were filtered with a first-order Savitzky-Golay filter. This filtering also helped to compensate for noise and tracking errors. In a next step, the data was downsampled to $50 \mathrm{~Hz}$. A total of 8,589 valid movement segments is extracted for both experiments.

\section{RESULTS}

In this section, we present the results for the previously explained HHI experiments. The details of the preprocessing steps, the features used for analysis, and the used machine learning method are provided. Next, we show that the proposed ontology is invariant to the positioning of the dyad for a subset of the ontology cases (H2 in Section 6.3). This is followed by case classification results for the full (20-case), and the reduced ontology (7-case), both of which verifies our first hypothesis that the ontology cases are distinguishable (H1 in Section 6.3). Subsequently, we briefly introduce results on the intrapersonal level. Lastly, we demonstrate how the ontology cases can be used to 
Table 1. Normalized Confusion Matrix for the Case Classification Accuracy with the Full Ontology

\begin{tabular}{|c|c|c|c|c|c|c|c|c|c|c|c|c|c|c|c|c|c|c|c|c|}
\hline & 1 & 2 & 3 & 4 & 8 & 14 & 15 & 16 & 20 & 5 & 6 & 9 & 13 & 19 & 7 & 11 & 10 & 12 & 17 & 18 \\
\hline 1 & 89.6 & 7.2 & 1.9 & 0.1 & 0.0 & 0.0 & 0.0 & 0.0 & 0.0 & 0.1 & 0.0 & 0.1 & 0.1 & 0.5 & 0.1 & 0.1 & 0.0 & 0.0 & 0.0 & 0.0 \\
\hline 2 & 8.7 & 80.9 & 6.4 & 0.6 & 0.2 & 0.0 & 0.9 & 0.0 & 0.1 & 1.0 & 0.1 & 0.3 & 0.2 & 0.7 & 0.0 & 0.0 & 0.0 & 0.0 & 0.0 & 0.0 \\
\hline 3 & 2.6 & 12.7 & 77.1 & 0.0 & 1.2 & 1.2 & 0.7 & 0.6 & 0.4 & 0.5 & 0.4 & 0.2 & 0.1 & 1.0 & 0.2 & 0.1 & 0.2 & 0.2 & 0.3 & 0.1 \\
\hline 4 & 1.0 & 12.6 & 0.2 & 70.0 & 6.4 & 0.3 & 8.4 & 0.0 & 1.0 & 0.0 & 0.0 & 0.0 & 0.0 & 0.0 & 0.1 & 0.0 & 0.0 & 0.0 & 0.0 & 0.0 \\
\hline 8 & 0.2 & 5.5 & 1.4 & 8.6 & 69.9 & 4.6 & 6.1 & 2.2 & 1.4 & 0.0 & 0.0 & 0.0 & 0.0 & 0.0 & 0.0 & 0.0 & 0.0 & 0.0 & 0.0 & 0.0 \\
\hline 14 & 0.1 & 0.5 & 7.7 & 0.0 & 3.8 & 63.9 & 3.2 & 11.5 & 8.7 & 0.0 & 0.0 & 0.0 & 0.0 & 0.0 & 0.5 & 0.0 & 0.0 & 0.0 & 0.0 & 0.0 \\
\hline 15 & 2.0 & 11.9 & 1.0 & 5.5 & 5.7 & 2.2 & 67.1 & 0.4 & 3.0 & 0.0 & 0.0 & 0.0 & 0.0 & 0.0 & 0.9 & 0.2 & 0.0 & 0.0 & 0.0 & 0.0 \\
\hline 16 & 0.0 & 1.8 & 6.6 & 0.0 & 2.5 & 14.4 & 2.6 & 61.1 & 10.9 & 0.0 & 0.0 & 0.0 & 0.0 & 0.0 & 0.1 & 0.0 & 0.0 & 0.0 & 0.0 & 0.0 \\
\hline 20 & 0.7 & 2.1 & 4.1 & 3.5 & 3.1 & 5.8 & 6.1 & 9.0 & 64.4 & 0.0 & 0.0 & 0.0 & 0.0 & 0.0 & 0.9 & 0.3 & 0.0 & 0.0 & 0.0 & 0.0 \\
\hline 5 & 0.4 & 2.1 & 0.0 & 0.0 & 0.0 & 0.0 & 0.0 & 0.0 & 0.0 & 56.2 & 3.6 & 15.9 & 18.2 & 2.1 & 0.0 & 0.0 & 0.4 & 0.1 & 0.6 & 0.5 \\
\hline 6 & 0.1 & 0.7 & 0.2 & 0.0 & 0.0 & 0.0 & 0.0 & 0.0 & 0.0 & 2.9 & 84.9 & 5.2 & 2.4 & 1.6 & 0.0 & 0.0 & 0.6 & 0.2 & 0.7 & 0.4 \\
\hline 9 & 0.7 & 1.2 & 1.6 & 0.0 & 0.0 & 0.0 & 0.0 & 0.0 & 0.0 & 19.4 & 4.5 & 51.9 & 14.5 & 2.5 & 0.0 & 0.0 & 1.0 & 0.9 & 1.4 & 0.4 \\
\hline 13 & 0.0 & 0.0 & 0.0 & 0.0 & 0.0 & 0.0 & 0.0 & 0.0 & 0.0 & 14.4 & 0.4 & 5.5 & 79.5 & 0.0 & 0.0 & 0.0 & 0.0 & 0.0 & 0.1 & 0.1 \\
\hline 19 & 4.3 & 13.0 & 1.0 & 0.0 & 0.0 & 0.0 & 0.0 & 0.0 & 0.1 & 2.5 & 1.7 & 2.3 & 1.2 & 73.4 & 0.0 & 0.0 & 0.1 & 0.0 & 0.3 & 0.0 \\
\hline 7 & 6.1 & 3.3 & 3.8 & 1.5 & 0.4 & 0.4 & 1.5 & 0.1 & 0.6 & 0.0 & 0.0 & 0.0 & 0.0 & 0.0 & 66.5 & 15.8 & 0.0 & 0.0 & 0.0 & 0.0 \\
\hline 11 & 1.5 & 3.6 & 4.5 & 1.3 & 0.5 & 0.5 & 0.8 & 0.7 & 0.9 & 0.0 & 0.0 & 0.0 & 0.0 & 0.0 & 18.0 & 67.7 & 0.0 & 0.0 & 0.0 & 0.0 \\
\hline 10 & 2.4 & 0.3 & 0.1 & 0.0 & 0.0 & 0.0 & 0.0 & 0.0 & 0.0 & 0.9 & 2.7 & 1.1 & 0.3 & 0.6 & 0.0 & 0.0 & 61.3 & 9.7 & 18.7 & 1.9 \\
\hline 12 & 1.0 & 0.7 & 0.3 & 0.0 & 0.0 & 0.0 & 0.0 & 0.0 & 0.0 & 0.1 & 3.2 & 1.0 & 0.0 & 0.0 & 0.0 & 0.0 & 22.5 & 51.6 & 11.1 & 8.3 \\
\hline 17 & 1.2 & 0.4 & 0.0 & 0.0 & 0.0 & 0.0 & 0.0 & 0.0 & 0.0 & 1.2 & 2.3 & 0.9 & 0.2 & 0.3 & 0.0 & 0.0 & 12.4 & 3.4 & 68.7 & 9.1 \\
\hline 18 & 0.2 & 0.3 & 0.1 & 0.0 & 0.0 & 0.0 & 0.0 & 0.0 & 0.0 & 1.4 & 5.2 & 1.2 & 1.6 & 0.0 & 0.0 & 0.0 & 6.3 & 7.9 & 32.5 & 43.4 \\
\hline
\end{tabular}

Order and grouping of the cases are according to the reduced ontology.

learn case-specific policies via training LSTM networks. These policies are transferred to a robotic agent to control its end-effector during similar interaction setups.

\subsection{Features}

It is first necessary to find a measure of the interactivity in order to classify interactions. Referring back to Section 3, the most important factors for the interaction are location and timing awareness, if the task is known. The location property during the interaction can be captured by the pairwise distances between kinematic features of agents. For a similar problem, Mainprice et al. (2016) used the pairwise distances between the recorded joint positions. However, this measure is not representative enough since it does not measure the shortest distance between the limbs, which could also have contact. Even the distance between the relevant joints is not close to a critical value, e.g., if forearms of agents cross each other orthogonally. Hence, we also compute the pairwise shortest distances between the limbs as additional features.

The total feature vector has a dimension of 25 . For the classification of each case, we used the mean feature values for each segment. The classification was conducted using a support vector machine (SVM) with a quadratic kernel. For training purposes, holdout validation was used.

\subsection{Location Invariance}

We used the data from the first experiment to validate the hypothesis $(\mathrm{H} 2)$ on positioning invariance. An SVM classifier is trained for the three ontology cases with a $50 \%$ holdout validation, each of which consisted of recordings from three different dyad positionings. We then computed the confusion matrix with respect to the prediction of each case, and the relative position used in the recording (Table 2). The recognition rates for each case are above 83\%. Furthermore, the 
Table 2. Normalized Confusion Matrix for the Location Invariance Analysis

\begin{tabular}{|c|c|c|c|c|c|c|c|c|c|}
\hline & \multicolumn{9}{|c|}{ Actual Case } \\
\hline & \multicolumn{3}{|c|}{1} & \multicolumn{3}{|c|}{2} & \multicolumn{3}{|c|}{3} \\
\hline & $\mathbf{F}$ & $\mathbf{O}$ & $\mathbf{S}$ & $\mathbf{F}$ & $\mathbf{O}$ & $\mathbf{S}$ & $\mathbf{F}$ & $\mathbf{O}$ & $\mathbf{S}$ \\
\hline$\stackrel{1}{e}$ & & 91 & & 3 & 3 & 2 & 1 & 1 & 1 \\
\hline ;亏 2 & 3 & 3 & 4 & & 83 & & 2 & 2 & 3 \\
\hline 3 & 1 & 1 & 2 & 4 & 5 & 5 & & 83 & \\
\hline
\end{tabular}

Predictions are further split into the tested dyad positions, frontal $(\mathrm{F})$, orthogonal $(\mathrm{O})$, same side $(\mathrm{S})$. The shaded-gray regions show the overall accuracy for the predicted case (total sum for all the positions (F, O, S) together for true classification). The remaining numbers indicate which position the actual case belongs to when it is misclassified.

Table 3. Normalized Confusion Matrix for the Interpersonal Test with the Reduced Ontology

\begin{tabular}{|c|c|c|c|c|c|c|c|}
\hline \multirow[b]{2}{*}{$\%$} & \multicolumn{7}{|c|}{ Predicted class } \\
\hline & 1 & 2 & 3 & 4 & 5 & 7 & 10 \\
\hline 1 & 89.6 & 6.9 & 1.8 & 0.2 & 1.2 & 0.2 & 0.1 \\
\hline 22 & 8.2 & 78.2 & 6.3 & 3.5 & 3.8 & 0.0 & 0.0 \\
\hline 3 & 2.6 & 13.0 & 76.7 & 4.3 & 2.3 & 0.3 & 0.8 \\
\hline$\vec{e}_{\mathrm{e}} 4$ & 0.7 & 3.1 & 2.1 & 93.6 & 0.0 & 0.5 & 0.0 \\
\hline 5 & 0.8 & 1.5 & 0.5 & 0.0 & 95.9 & 0.0 & 1.2 \\
\hline 7 & 2.8 & 2.6 & 2.5 & 6.9 & 0.0 & 85.2 & 0.0 \\
\hline 10 & 0.9 & 0.0 & 0.0 & 0.0 & 6.7 & 0.0 & 92.4 \\
\hline
\end{tabular}

misclassified segments are evenly distributed among the relative positions, which indicates that no relative position has a strong influence on the misclassification rate. Consequently, those ontology cases are location invariant w.r.t. the features used, supporting the first hypothesis $(H 1$ in Section 6.3) we investigate.

\subsection{Ontology Classification}

Classification accuracy for all ontology cases were assessed by using the data of all participants and randomly splitting them into two holds of 50\% data size. In a next step, we trained a quadratic SVM for 100 times and computed the normalized confusion matrix both for the full ontology (20-case, Table 1), as well as the reduced ontology (7-case, Table 3). The confusion matrix of the full ontology is sorted by the respective merged cases, for which the overall recognition rate of $74 \%$ is achieved. Considering the block diagonal structure of the correct and incorrect recognition rates, the merged cases cover most of the misclassifications. This is further confirmed in the confusion matrix of the reduced ontology, for which the overall recognition rate increases to $88 \%$. Furthermore, the remaining highest misclassifications mostly result from cases 2 and 3, with the rest having a recognition rate above $85 \%$. These classification rates support our hypothesis ( $H 1$ in Section 6.3) that, with the selected distance-based features, interaction cases can be identified with high accuracy. Put differently, there are unique behavioral patterns depending on the interaction setup that justify our top-down construction of graph-based ontology cases. 
Table 4. Results of Intrapersonal Movement Behavior Classification Tests

\begin{tabular}{lcccccc}
\hline Subject\# & $\mathbf{1}$ & $\mathbf{2}$ & $\mathbf{3}$ & $\mathbf{4}$ & $\mathbf{5}$ & $\mathbf{6}$ \\
\hline Intra-Subject & 91.0 & 89.2 & 91.7 & 92.4 & 92.9 & 93.5 \\
Subj. vs. Group & 67.4 & 38.3 & 46.8 & 65.3 & 63.8 & 61.6 \\
Group vs. Subj. & 66.0 & 67.7 & 69.6 & 83.5 & 85.7 & 76.4 \\
\hline
\end{tabular}

First row: testing intra-subject, second row: subject versus rest, third row rest versus subject.

Table 5. Summary of Training and Test Data Separation

\begin{tabular}{lcccc}
\hline Trajectories & Case 1 & Case 2 & Case 3 & Generic Model \\
\hline Training & 5,348 & 5,104 & 4,904 & 32,632 \\
Test & 70 & 68 & 65 & 429 \\
\hline
\end{tabular}

\subsection{Intrapersonal Classification}

For the intrapersonal classification, we could use the data of six participants who performed both experiments. All of them were male of age $25 \pm 2$ years. The classification was conducted using the reduced ontology with seven cases. The results of each person vary from 89.2 to $93.5 \%$ (Table 4, first row), which is comparable to the results from the complete dataset. By testing the classifiers from each individual person against the rest of the data, we evaluated the generalization of individual behaviors to the overall behavioral patterns. These recognition rates range from 38.3 to $67.4 \%$ (Table 4, second row), which are significantly less than the overall and intrapersonal classification results. On the one hand, individual behavior generalizes up to some degree, especially in cases around 65\% recognition rates, which are above chance. However, on the other hand, each individual person still has distinct behavioral patterns during interaction. This argument is further supported by tests, in which the individual data is classified with the model trained on the rest of the data (Table 4, third row), for which the recognition rates range from 66.0 to $85.7 \%$. This improvement compared to the individual rates indicates that a broader range of behaviors is able to generalize quite well, and that most behaviors fall into this generalization.

\subsection{Case-Specific Policies with LSTMs}

Our recent work has shown that imitation learning for close proximity HRI is feasible by replicating human interaction behavior (Oguz et al. 2018a). However, ontology cases have not been utilized in this previous study. In order to check whether the ontology is capable of representing different interaction behaviors, control models for each case were learned from data individually. Those models are learned as RNN-based policies, specifically, as LSTM units, which are introduced in Section 5. By comparing case-specific LSTM-based policies, which have been trained only with the data from the corresponding case, to the single generic policy, which has been trained on the whole set of data, one key benefit of the ontology is established. In essence, human hand movement behavior is captured better with the case-specific policies.

A summary of the dataset used to train each of the models, and the size of the test sets in each case are presented in Table 5. The LSTM policy architecture was configured as a 2-layer, each with 32 hidden units, network. For this analysis, sample trajectories generated by using either the generic or the case-specific control policies are compared with the recorded human hand movement trajectory for scenarios being evaluated. The first three cases were investigated for this analysis. Dynamic time warping (DTW) algorithm, which measures similarity between two temporal 


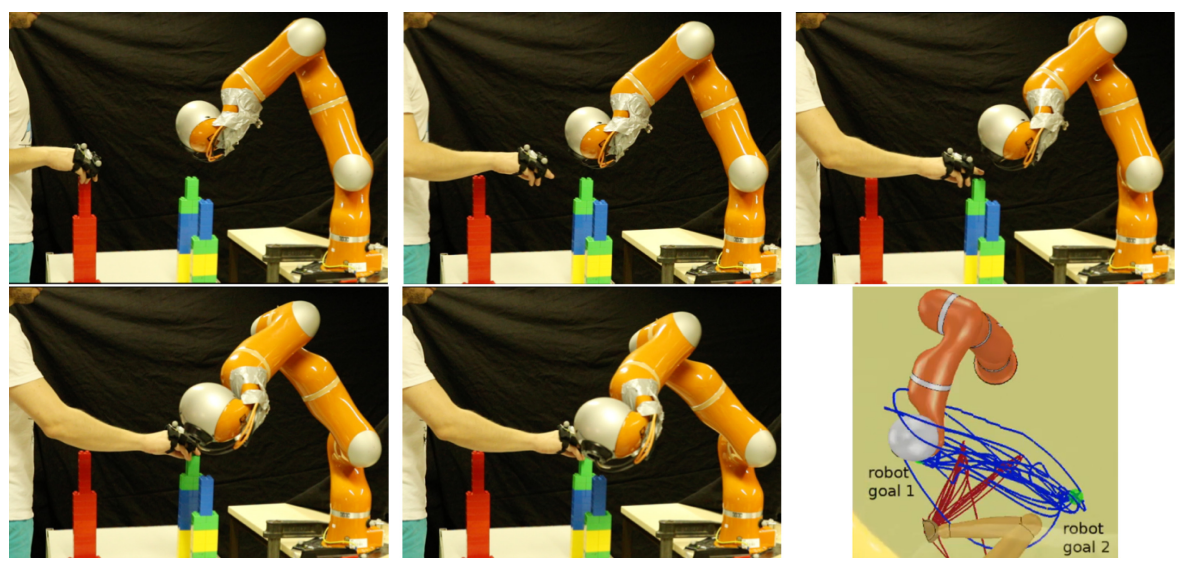

Fig. 6. The sequence of scenes from a close proximity HRI with a Kuka robot, and the top-down view of the trajectories of the human hand and the robot end-effector (lower-right) (see supplementary video).

sequences by calculating an optimal match between them w.r.t. some distance measure, is used for the comparison. In particular, a Euclidean distance measure is used and the DTW cost is reported with respect to the reference human trajectory from the dataset in each case. We also consider two types of output layers, MSE GMM.

The DTW scores indicate that, both for the particular test sample and the average of the test set, case-specific control policy produces interaction trajectories that are spatially closer to the human samples in the test set for cases 1,2, and 3 (Figure 7 and Table 6). The behavior holds for MSE and GMM output layer policies, with the latter producing slightly better generic control policy performance, but suffers from convergence to the goal position. This indicates that the ontology with its distinct interaction cases enables learning respective unique movement and control patterns. Hence, autonomous agents can be equipped with complex control and motion planning capabilities to assist human partners depending on the collaboration setup requirements.

7.5.1 Transfer to a Robot. The spatial comparison analysis, presented in the previous section, was conducted in a simulated environment, where the human partner's movement was rolled out from the recorded data, and the learned policies were used to control a robotic end-effector position. In addition, we also constructed a proof-of-concept, real-world HRI setup using the LSTM policies. The end-effector of a KUKA LWR4+ was controlled by the learned policy, while a damped least-squares IK solver was used for the joint positions, in a close proximity HRI setup (Figure 6). Even though the setup is similar to the ontology case 2, the task parameters, i.e., the action locations for the robotic agent, were different than the human-human interaction data used for training the policy. Still, the robot was successful at executing point-to-point reaching movements closer to a human partner.

The policy has also been tested for various other tasks in order to evaluate its capabilities further (Figure 8). It has been found out that the policy enables the robot to execute a sequence of tasks smoothly (note that the training did not involve any explicit guidance on stopping and then restarting movement for another task). More notably, the policy was tested for goal switching in mid-flight, in an attempt to simulate a task selection due to some interruption, e.g., human partner blocking the path. The robot manages to change the task, with a distance-based rule, and successfully reaches the new target location without interruption in motion. Lastly, we tested whether the 


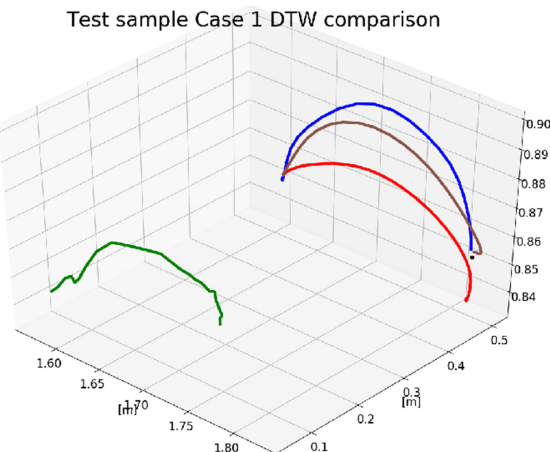

Test sample Case 2 DTW comparison

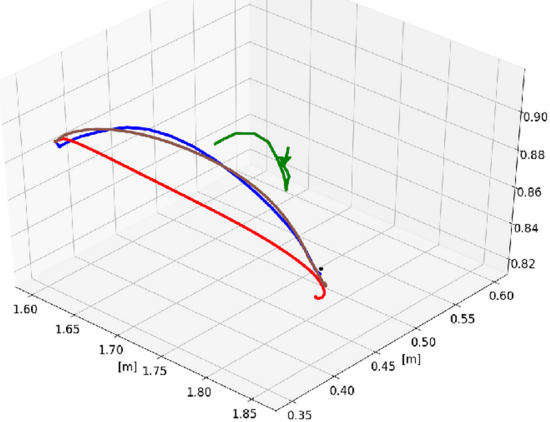

Test sample Case 3 DTW comparison

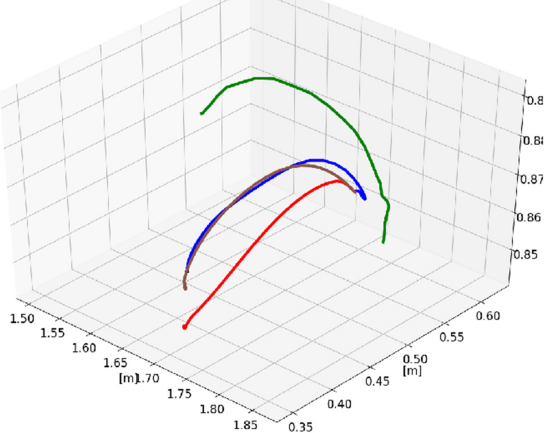

Test sample Case 1 DTW comparison

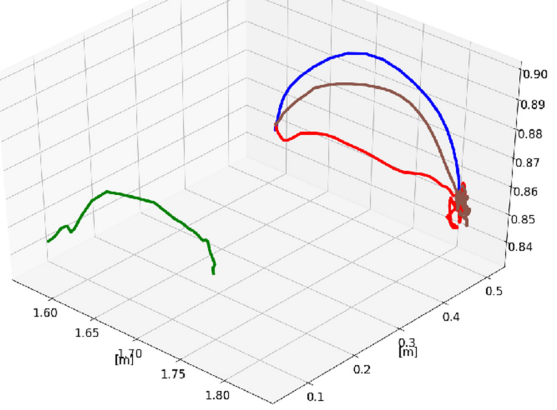

Test sample Case 2 DTW comparison

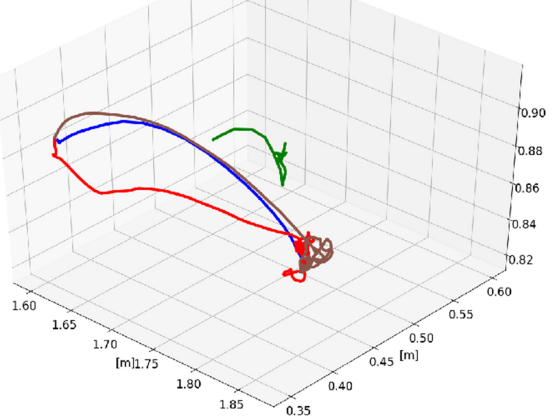

Test sample Case 3 DTW comparison

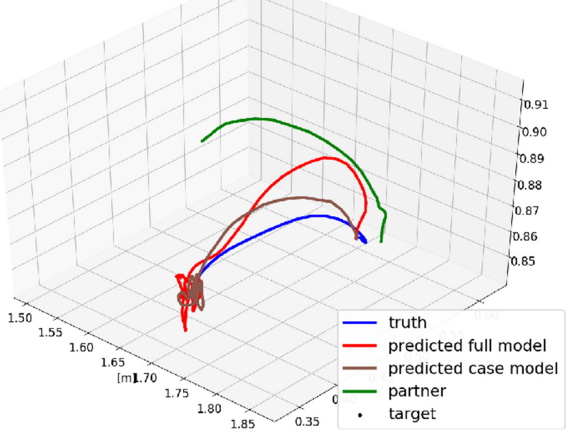

Fig. 7. The DTW comparison of exemplary trajectories generated by generic and case-specific models: case 1,2 , and 3 (top, middle, bottom, respectively); MSE and GMM output layers (left, right, respectively).

Table 6. Summary of DTW Scores for Tested Models (Smaller is Better)

\begin{tabular}{lccc}
\hline Models & Case 1 & Case 2 & Case 3 \\
\hline generic mse & 4.1269 & 4.4552 & 3.9024 \\
case mse & 2.1020 & 3.2260 & 2.2369 \\
generic gmm & 2.9839 & 3.3552 & 2.8199 \\
case gmm & 2.6176 & 3.1067 & 2.4782 \\
\hline
\end{tabular}




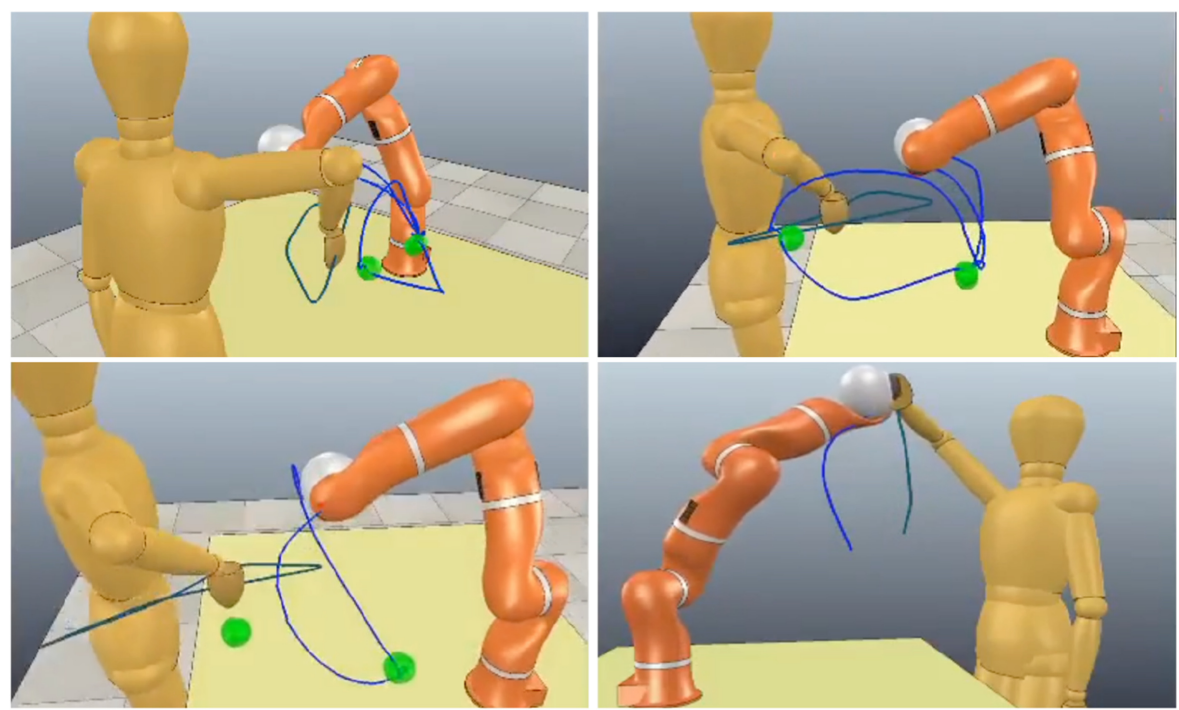

Fig. 8. The scenes from a close proximity HRI with a Kuka robot (the real robot was controlled simultaneously, but for better explaining, the interactions with visual, simulated versions are used). The sequential task execution (top row, different perspectives), task change due to possible collision with a human (bottom-left), and handover/high-5-like scenario where the robot follows a dynamic target (bottom-right) with human hand trajectories (dark green), robot end-effector trajectories (blue), and robot target positions (green).

robot could follow a moving target to simulate a handover scenario, and again, the robot was able to reach a moving target swiftly. All of those capabilities are shown in the supplementary video.

\section{DISCUSSION}

In this section, a further analysis and discussion of our results is presented. The results from the classification analysis have provided multiple insights into the dyadic interaction behavior control. First, the features used can adequately describe and distinguish different interaction scenarios. Second, we could show that the construction of the full ontology is reasonable, since each case is recognized with a rate of at least $45 \%$, which is $40 \%$ points above chance, and overall $74 \%$. Furthermore, the analysis on the full ontology confirmed that merging some cases with a top down approach is feasible due to their similarity in spatial features. The resulting reduced ontology has also proven to be effective, increasing the overall recognition rate up to $88 \%$. It has to be noted that there also exist different feasible mergers, as it can be seen in the confusion matrix of the full ontology. Nevertheless, we picked the most effective combinations based on our systematic deduction and data analysis.

The results from the intrapersonal analysis also support the feasibility of the reduced ontology. The high average intrapersonal recognition rates of $91.8 \%$ confirm that there exist distinct interaction scenarios that demand different dyadic movement behaviors. Still, these interaction scenarios also depend strongly on individual behavior, as it can be seen in the group comparison results. However, this individual behavior is still generalizable for each ontology case, based on the generalization results, as well as the interpersonal results.

For the ontology cases 1,2, and 3, we have shown that those cases are invariant to the dyad positioning we considered (Table 3). Despite only a subset of the ontology cases being tested, we 
assume that this either holds true for the rest as well, or for some cases is infeasible to evaluate. This assumption is based on the fact that the tested cases are the least restricted of the whole ontology. For instance, there is no shared workspace (case 13), or necessary specific setup (case 10), or restrictions of the motion of one participant (case 8) in some cases. Hence, we assume that a dependence on the positioning of the dyad, if it exists, would be visible in those cases with high degrees of freedom. Since such a difference was found to be small, the variance due to the positioning of the dyad w.r.t. the features used for representing them is assumed to be negligible for other cases as well.

In addition to the location invariance for the first three cases, with the second experiment, we have shown that the ontology is also invariant to small setup changes, as slight variations on the action spaces were intentionally enforced. This is further supported by the better classification results of the merged ontology. Additionally, the proposed ontology is claimed to be task invariant and, as such, usable in a multitude of scenarios. This is presumed valid due to the fact that most manipulation tasks can be split into pick and place tasks (Wörgötter et al. 2013). The only restriction on this assumption should be the actions on objects that dynamically influence the motion of the actor, i.e., comparably bigger objects. The ontology framework can also generalize to joint tasks, such as handover tasks, or joint assembly tasks. This can be realized by using cases with a shared workspace (e.g., case 13), while enforcing the synchronicity of the dyad behavior, as shown in simulation of an interaction between human and robot.

Besides classifying human motion behavior, we also tried to verify if humans tend to synchronize their movements in different setups. We could observe the tendency to synchronization during the experiments. However, we were unable to show this effect in our synchronization estimate. We attribute this to the difficulty to measure synchronization for tasks, in which the motion trajectories are intersecting, as well as for tasks that allow a variance of end-effector poses at each action space, all of which impose interaction variations specific to the case. Based on these insights, and the option of using timing to enforce behaviors such as handover tasks, we believe that timing of robotic actions and movements might be used as an active control input instead of using it as a descriptive element or metric.

Our analysis on the learned policies and their transfer to a real robot highlighted some critical points. First, the feasibility of learning control policies from HHI data is verified. Second, ontology cases enable specifying effective case-specific controllers, which might be utilized for constructing complex behaviors, e.g., with a hierarchical structure, rather than relying on a single motion planner for collaborative robots. Lastly, generalization capability of those imitated policies is also observed when tested on unknown scenarios.

It is worth noting a few critical issues. For instance, a direct transfer of policies learned only from human movement data is not sufficient to achieve safe and effective close proximity HRI. For instance, we can not guarantee robustness and convergence properties for the LSTM-based policies, which, at its current form, also prevents us to conduct user studies. This is also partially related to the discrepancies in human interaction behavior while working alongside a real robot. Still, such imitation policies offer feasible initial controllers, which can be adjusted with a policy improvement algorithm during interaction by the autonomous agent to take into account the observed human behaviors and the team fluency. Another bottleneck is the kinematic difference between a human and a robot. Only the hand movement control is imitated in this work, and a damped least-square inverse kinematics solver is used for joint control. Although imitating the human hand movement characteristics provides an essential and suitable behavior for natural dyadic interaction, further research is necessary to extend our work for fully controlling all the joints of an autonomous agent. 


\section{CONCLUSION AND FUTURE WORK}

In this work, we presented an ontology for HHI for a shared workspace scenario. We derived an interaction model based on insights from psychology and neuroscience, and used that model to derive the ontology. This ontology was analyzed in depth, and a reduction of the ontology cases was proposed. It has been shown that the ontology cases can be recognized with high accuracy. Moreover, we have shown that the ontology generalizes well to different behaviors, and it can partially handle unknown behaviors. Invariance to dyad positioning for a subset of ontology cases has also been demonstrated. Lastly, unique interaction policies, that a robotic agent can use to generate interaction motions, have been learned for each case. In essence, the proposed ontology is a first step toward understanding close proximity collaboration behaviors in detail and transferring human-like dyadic movement control policies to robotic agents for achieving natural, safe, and effective human-robot collaboration.

This study not only encourages but also provides a generic procedure for systematically exploring non-physical, close proximity interaction movement behaviors and building control policies for dyadic interaction in subsequent studies. Such a thorough coverage and analysis of HHI cases will serve as a base and reference for later studies to propose and benchmark new learning algorithms, and evaluate close proximity HRI behaviors for a wide range of scenarios.

In essence, several research fields provided us with effective tools and critical insights to investigate close-proximity HHI from a top-down perspective, which resulted in the proposed ontology cases. In addition, using a data-driven approach allowed us not only to explore dyadic movements with a bottom-up approach, but also to construct controllers for autonomous agents that interact with humans. We believe such bidirectional approaches are fundamental for realizing generalizable planners that enable effective, natural, and safe HRI in close proximity.

Even though the learned policies were successfully applied on a real robot and tested during a close proximity human-robot interaction setup as a proof-of-concept, the safety of the human is not guaranteed. In future work, these learned control policies can be extended with additional sensory information and control mechanisms to provide a safer yet still natural HRI in a shared workspace. Only then, an experiment with human subjects would show if the ontology can directly be transferred to HRI, or whether human partners exhibit a different behavior during HRI. In case human interaction behavior variates from the previously learned control models for each interaction case, a policy improvement algorithm can be utilized to adapt the policy parameters quickly w.r.t. the human partner movement. Furthermore, it would be useful to test the ontology with different tasks in order to verify the proposed task invariance further, and also to extend it to more agents to demonstrate the effectiveness of the proposed graph-based method.

\section{REFERENCES}

Henny Admoni, Anca D. Dragan, Siddhartha S. Srinivasa, and Brian Scassellati. 2014. Deliberate delays during robot-tohuman handovers improve compliance with gaze communication. In ACM/IEEE International Conference on HumanRobot Interaction, HRI'14, Bielefeld, Germany, March 3-6, 2014. ACM, 49-56. DOI: https://doi.org/10.1145/2559636. 2559682

Mohamad Javad Aein, Eren Erdal Aksoy, Minija Tamosiunaite, Jeremie Papon, Ales Ude, and Florentin Wörgötter. 2013. Toward a library of manipulation actions based on semantic object-action relations. In 2013 IEEE/RSf International Conference on Intelligent Robots and Systems. IEEE, 4555-4562.

Eren Erdal Aksoy, Alexey Abramov, Johannes Dörr, Kejun Ning, Babette Dellen, and Florentin Wörgötter. 2011. Learning the semantics of object-action relations by observation. Int. F. Rob. Res. 30, 10 (2011), 1229-1049.

Eren Erdal Aksoy, Minijia Tamosiunaite, Rok Vuga, Ales Ude, C. Geib, Mark Steedman, and Florentin Worgotter. 2013. Structural bootstrapping at the sensorimotor level for the fast acquisition of action knowledge for cognitive robots. In IEEE 3rd foint International Conference on Development and Learning and Epigenetic Robotics (ICDL'13). IEEE, 1-8. 
A. Alahi, K. Goel, V. Ramanathan, A. Robicquet, L. Fei-Fei, and S. Savarese. 2016. Social LSTM: Human trajectory prediction in crowded spaces. In IEEE Conf. on Comp. Vision and Pattern Rec. (CVPR). 961-971. DOI : https://doi.org/10.1109/CVPR. 2016.110

Telmo Amaral, Luís M. Silva, Luís A. Alexandre, Chetak Kandaswamy, Joaquim Marques de Sá, and Jorge M. Santos. 2014. Transfer learning using rotated image data to improve deep neural network performance. In Int. Conf. Image Anal. and Recog., Portugal, Oct. 22-24, 2014, Vol. 8814. Springer, 290-300. DOI : https://doi.org/10.1007/978-3-319-11758-4_32

Oliver Brock and Oussama Khatib. 2002. Elastic strips: A framework for motion generation in human environments. Int. 7 . Rob. Res. 21, 12 (2002), 1031-1052.

Maya Cakmak, Siddhartha S Srinivasa, Min Kyung Lee, Sara Kiesler, and Jodi Forlizzi. 2011. Using spatial and temporal contrast for fluent robot-human hand-overs. In Proceedings of the 6th International Conference on Human-Robot Interaction. ACM, 489-496.

Sylvain Calinon, Paul Evrard, Elena Gribovskaya, Aude Billard, and Abderrahmane Kheddar. 2009. Learning collaborative manipulation tasks by demonstration using a haptic interface. In International Conference on Advanced Robotics (ICAR'09). IEEE, 1-6.

Maurizio Corbetta and Gordon L. Shulman. 2002. Control of goal-directed and stimulus-driven attention in the brain. Nat. Rev. Neurosci. 3, 3 (2002), 201-215.

Robert Desimone and John Duncan. 1995. Neural mechanisms of selective visual attention. Ann. Rev. Neurosci. 18, 1 (1995), 193-222.

Philine Donner and Martin Buss. 2016. Cooperative swinging of complex pendulum-like objects: Experimental evaluation. IEEE Trans. Rob. 32, 3 (2016), 744-753.

Aaron Edsinger and Charles C. Kemp. 2007. Human-robot interaction for cooperative manipulation: Handing objects to one another. In RO-MAN 2007-The 16th IEEE International Symposium on Robot and Human Interactive Communication. IEEE, 1167-1172.

Howard E. Egeth and Steven Yantis. 1997. Visual attention: Control, representation, and time course. Ann. Rev. Psychol. 48, 1 (1997), 269-297.

Charles L. Folk, Roger W. Remington, and James C. Johnston. 1992. Involuntary covert orienting is contingent on attentional control settings. F. Exp. Psychol. Hum. Percept. Perform. 18, 4 (1992), 1030-1044.

Dashan Gao, Vijay Mahadevan, and Nuno Vasconcelos. 2008. On the plausibility of the discriminant center-surround hypothesis for visual saliency. F. Vision 8, 7 (2008), 13-13.

Rafi Hayne, Ruikun Luo, and Dmitry Berenson. 2016. Considering avoidance and consistency in motion planning for human-robot manipulation in a shared workspace. In IEEE International Conference on Robotics and Automation (ICRA'16). IEEE, 3948-3954.

Guy Hoffman. 2013. Evaluating fluency in human-robot collaboration. In International Conference on Human-Robot Interaction (HRI), Workshop on Human Robot Collaboration, Vol. 381. 1-8.

Guy Hoffman and Cynthia Breazeal. 2007. Effects of anticipatory action on human-robot teamwork efficiency, fluency, and perception of team. In Proceedings of the ACM/IEEE International Conference on Human-robot Interaction. ACM, 1-8.

Laurent Itti and Christof Koch. 2001. Computational modelling of visual attention. Nat. Rev. Neurosci. 2, 3 (2001), $194-203$.

Ashesh Jain, Amir R. Zamir, Silvio Savarese, and Ashutosh Saxena. 2016. Structural-RNN: Deep learning on spatio-temporal graphs. In Proceedings of the IEEE Conference on Computer Vision and Pattern Recognition. 5308-5317.

Günther Knoblich and Rüdiger Flach. 2001. Predicting the effects of actions: Interactions of perception and action. Psychol. Sci. 12, 6 (2001), 467-472.

Hema Koppula and Ashutosh Saxena. 2013. Learning spatio-temporal structure from rgb-d videos for human activity detection and anticipation. In Proceedings of the 30th International Conference on Machine Learning (ICML-13). 792-800.

Hema Swetha Koppula, Rudhir Gupta, and Ashutosh Saxena. 2013. Learning human activities and object affordances from rgb-d videos. Int. F. Rob. Res. 32, 8 (2013), 951-970.

Hema S Koppula and Ashutosh Saxena. 2014. Physically grounded spatio-temporal object affordances. In European Conference on Computer Vision. Springer, 831-847.

Claus Lenz, Suraj Nair, Markus Rickert, Alois Knoll, Wolgang Rosel, Jurgen Gast, Alexander Bannat, and Frank Wallhoff. 2008. Joint-action for humans and industrial robots for assembly tasks. In The 17th IEEE International Symposium on Robot and Human Interactive Communication (RO-MAN'08). IEEE, 130-135.

Yanan Li, Keng Peng Tee, Wei Liang Chan, Rui Yan, Yuanwei Chua, and Dilip Kumar Limbu. 2015. Role adaptation of human and robot in collaborative tasks. In IEEE International Conference on Robotics and Automation (ICRA'15). IEEE, 5602-5607.

Tamara Lorenz, Björn NS Vlaskamp, Anna-Maria Kasparbauer, Alexander Mörtl, and Sandra Hirche. 2014. Dyadic movement synchronization while performing incongruent trajectories requires mutual adaptation. Frontiers in Human Neuroscience 8 (2014), 461. 
Jim Mainprice, Rafi Hayne, and Dmitry Berenson. 2015. Predicting human reaching motion in collaborative tasks using inverse optimal control and iterative re-planning. In 2015 IEEE International Conference on Robotics and Automation (ICRA). IEEE, 885-892.

Jim Mainprice, Rafi Hayne, and Dmitry Berenson. 2016. Goal set inverse optimal control and iterative replanning for predicting human reaching motions in shared workspaces. IEEE Trans. Rob. 32, 4 (2016), 897-908.

S. Murata, Y. Li, H. Arie, T. Ogata, and S. Sugano. 2018. Learning to achieve different levels of adaptability for humanrobot collaboration utilizing a neuro-dynamical system. IEEE Trans. Cog. Dev. Syst. 10, 3 (Sept. 2018), 712-725. DOI : https://doi.org/10.1109/TCDS.2018.2797260

Bilge Mutlu, Allison Terrell, and Chien-Ming Huang. 2013. Coordination mechanisms in human-robot collaboration. In Proceedings of the Workshop on Collaborative Manipulation, 8th ACM/IEEE International Conference on Human-Robot Interaction. Citeseer, 1-6.

Duc-Canh Nguyen, Gérard Bailly, and Frédéric Elisei. 2017. Learning off-line vs. on-line models of interactive multimodal behaviors with recurrent neural networks. Pattern Recognit. Lett. 100 (2017), 29-36. DOI : https://doi.org/10.1016/j.patrec. 2017.09.033

Ozgur S. Oguz, Ayse Kucukyilmaz, T. Metin Sezgin, and Cagatay Basdogan. 2012. Supporting negotiation behavior with haptics-enabled human-computer interfaces. IEEE Trans. Haptics 5, 3 (2012), 274-284. DOI : https://doi.org/10.1109/TOH. 2012.37

O. S. Oguz, B. M. Pfirrmann, M. Guo, and D. Wollherr. 2018a. Learning hand movement interaction control using RNNs: From HHI to HRI. IEEE Rob. Autom. Lett. 3, 4 (Oct 2018), 4100-4107. DOI : https://doi.org/10.1109/LRA.2018.2862923

Ozgur S. Oguz, Omer C. Sari, Khoi H. Dinh, and Dirk Wollherr. 2017. Progressive stochastic motion planning for humanrobot interaction. In 2017 26th IEEE International Symposium on Robot and Human Interactive Communication (RO-MAN).

Ozgur S. Oguz, Zhehua Zhou, and Dirk Wollherr. 2018b. A hybrid framework for understanding and predicting human reaching motions. Front. Rob. AI 5 (2018), 27. DOI : https://doi.org/10.3389/frobt.2018.00027

Chonhyon Park, Jia Pan, and Dinesh Manocha. 2013. Real-time optimization-based planning in dynamic environments using GPUs. In IEEE International Conference on Robotics and Automation (ICRA'13). IEEE, 4090-4097.

Mark Pfeiffer, Giuseppe Paolo, Juan I. Nieto, Roland Siegwart, and Cesar Cadena. 2017. A data-driven model for interactionaware pedestrian motion prediction in object cluttered environments. In Proceedings of the IEEE International Conference on Robotics and Automation (ICRA'18). 1-8. https://doi.org/10.1109/ICRA.2018.8461157

Leonel Rozo Castañeda, Sylvain Calinon, Darwin Caldwell, Pablo Jimenez Schlegl, and Carme Torras. 2013. Learning collaborative impedance-based robot behaviors. In Proceedings of the 27th AAAI Conference on Artificial Intelligence. 14221428 .

Jill Sardegna, Susan Shelly, and Scott Steidl. 2002. The Encyclopedia of Blindness and Vision Impairment. Infobase Publishing.

Natalie Sebanz and Guenther Knoblich. 2009. Prediction in joint action: What, when, and where. Top. Cognit. Sci. 1, 2 (2009), 353-367.

Julie Shah, James Wiken, Brian Williams, and Cynthia Breazeal. 2011. Improved human-robot team performance using Chaski, a human-inspired plan execution system. In Proceedings of the 6th International Conference on Human-Robot Interaction. ACM, 29-36.

Kyle Wayne Strabala, Min Kyung Lee, Anca Diana Dragan, Jodi Lee Forlizzi, Siddhartha Srinivasa, Maya Cakmak, and Vincenzo Micelli. 2013. Towards seamless human-robot handovers. F. Hum.-Rob. Interact. 2, 1 (2013), 112-132.

M.P.S. To, B.C. Regan, Dora Wood, and J.D. Mollon. 2011. Vision out of the corner of the eye. Vision Res. 51, 1 (2011), 203-214.

Paul D. Tynan and Robert Sekuler. 1982. Motion processing in peripheral vision: Reaction time and perceived velocity. Vision Res. 22, 1 (1982), 61-68.

Emre Ugur, Yukie Nagai, Erol Sahin, and Erhan Oztop. 2015. Staged development of robot skills: Behavior formation, affordance learning and imitation with motionese. IEEE Tran. Auton. Ment.Dev. 7, 2 (2015), 119-139.

Emre Ugur and Justus Piater. 2015. Bottom-up learning of object categories, action effects and logical rules: From continuous manipulative exploration to symbolic planning. In IEEE International Conference on Robotics and Automation (ICRA).

Anirudh Vemula, Katharina Mülling, and Jean Oh. 2017. Social attention: Modeling attention in human crowds. In Proceedings of the IEEE International Conference on Robotics and Automation (ICRA'18). 1-7. https://doi.org/10.1109/ICRA.2018. 8460504

Katsumi Watanabe. 2008. Behavioral speed contagion: Automatic modulation of movement timing by observation of body movements. Cognition 106, 3 (2008), 1514-1524.

Florentin Wörgötter, Eren Erdal Aksoy, Norbert Krüger, Justus Piater, Ales Ude, and Minija Tamosiunaite. 2013. A simple ontology of manipulation actions based on hand-object relations. IEEE Trans. Auton. Ment. Dev. 5, 2 (2013), 117-134.

Received April 2018; revised December 2018; accepted April 2019 